\title{
Global subanalytic solutions of Hamilton-Jacobi type equations
}

\author{
Emmanuel Trélat \\ Univ. Paris-Sud, Labo. AN-EDP, Math., UMR 8628, Bat. 425, 91405 Orsay cedex, France
}

Received 14 February 2005; received in revised form 31 March 2005; accepted 11 May 2005

Available online 7 December 2005

\begin{abstract}
In the 80's, Crandall and Lions introduced the concept of viscosity solution, in order to get existence and/or uniqueness results for Hamilton-Jacobi equations. In this work, we first investigate the Dirichlet and Cauchy-Dirichlet problems for such equations, where the Hamiltonian is associated to a problem of calculus of variations, and prove that, if the data are analytic, then the viscosity solution is moreover subanalytic. We then extend this result to Hamilton-Jacobi equations stemming from optimal control problems, in particular from sub-Riemannian geometry, which are generalized eikonal equations.

As a consequence, the set of singularities of the viscosity solutions of such Hamilton-Jacobi equations is a subanalytic stratified manifold of codimension greater than or equal to one.

(ㄷ) 2006 L'Association Publications de l'Institut Henri Poincaré. Published by Elsevier B.V. All rights reserved
\end{abstract}

MSC: 49L25; 32B20

Keywords: Hamilton-Jacobi equation; Value function; Viscosity solution; Subanalytic sets

\section{Introduction}

\subsection{Viscosity solutions}

In the 80's, Crandall and Lions [20] introduced the concept of viscosity solution in order to ensure uniqueness of solutions of Hamilton-Jacobi equations. Existence of viscosity solutions was also established under similar assumptions. A general definition of a viscosity solution of a first-order Hamilton-Jacobi equation is the following.

Let $\Omega$ be an open set in $\mathbb{R}^{n}, H$ be a continuous function on $\Omega \times \mathbb{R} \times \mathbb{R}^{n}$, called Hamiltonian, and $g$ be a continuous function on $\partial \Omega$. Consider the first-order Hamilton-Jacobi equation on $\Omega$

$$
H(x, v(x), \nabla v(x))=0 .
$$

We first recall the notion of sub- and super-differential.

Definition 1.1. Let $v$ be a scalar function on $\Omega$. The super-differential at a point $x \in \Omega$ is defined as

$$
D^{+} v(x)=\left\{p \in \mathbb{R}^{n} \mid \limsup _{y \rightarrow x} \frac{v(y)-v(x)-\langle p, y-x\rangle}{\|y-x\|} \leqslant 0\right\} .
$$

E-mail address: emmanuel.trelat@math.u-psud.fr (E. Trélat). 
Similarly, the sub-differential at $x$ is

$$
D^{-} v(x)=\left\{p \in \mathbb{R}^{n} \mid \liminf _{y \rightarrow x} \frac{v(y)-v(x)-\langle p, y-x\rangle}{\|y-x\|} \geqslant 0\right\} .
$$

We can now define the concept of viscosity solution introduced in [20].

Definition 1.2. Let $v$ be a continuous function on $\Omega$. The function $v$ is a viscosity super-solution of (1) if

$$
\forall x \in \Omega \forall p \in D^{-} v(x) \quad H(x, v(x), p) \geqslant 0 .
$$

Similarly, $v$ is a viscosity sub-solution of (1) if

$$
\forall x \in \Omega \forall p \in D^{+} v(x) \quad H(x, v(x), p) \leqslant 0 .
$$

Finally, $v$ is a viscosity solution of (1) if it is both a sub-solution and a super-solution.

This concept is adapted to get existence and uniqueness results, in particular for Dirichlet problems of the type

$$
\begin{aligned}
& H(x, v(x), \nabla v(x))=0 \quad \text { in } \Omega, \\
& v_{\mid \partial \Omega}=g,
\end{aligned}
$$

so as for many other problems (Cauchy problems, second-order equations, ...), see for instance [20,27,9,10,21]. Literature on this subject is immense.

Viscosity solutions, when they exist, may be just continuous. Hence, the study of the regularity of such solutions is of great interest. Usually, regularity results are sought for in special classes of nonsmooth functions, such as Lipschitz or semiconcave functions (see for instance [14,15,31], and more generally, see the books [9,10,16,27] and references therein). In the case of analytic Hamilton-Jacobi equations, one could however expect these solutions to be more regular. Of course, because of possible shocks, one cannot expect to get global analytic solutions. For example, in the case of the eikonal equation

$$
\begin{aligned}
& \|\nabla v(x)\|^{2}=1 \quad \text { in } \Omega, \\
& v_{\mid \partial \Omega}=0,
\end{aligned}
$$

on a bounded analytic open set $\Omega \subset \mathbb{R}^{n}$, one can easily see that the unique viscosity solution is

$$
v(x)=d(x, \partial \Omega) .
$$

Of course, this function $u$ is not analytic on $\Omega$, due to intersection of characteristic curves (concerning the method of characteristics we refer the reader to the previously cited references). Anyway, the function $v$ is, in a sense, "analytic by parts". The right concept in order to describe such objects happens to be the concept of subanalyticity. In the next paragraph we recall a definition and several basic properties.

\subsection{Subanalytic functions}

We first recall a definition of subanalytic sets (see [23,24]).

Definition 1.3. Let $M$ be a real analytic finite dimensional manifold. A subset $A$ of $M$ is said to be semi-analytic if and only if, for every $x \in M$, there exists a neighborhood $U$ of $x$ in $M$ and $2 p q$ analytic functions $g_{i j}, h_{i j}(1 \leqslant i \leqslant p$ and $1 \leqslant j \leqslant q$ ), such that

$$
A \cap U=\bigcup_{i=1}^{p}\left\{y \in U \mid g_{i j}(y)=0 \text { and } h_{i j}(y)>0, j=1, \ldots, q\right\} .
$$

Let $\operatorname{SEM}(M)$ denote the set of semi-analytic subsets of $M$.

The image of a semi-analytic subset by a proper analytic mapping is not in general semi-analytic, and thus this class has to be enlarged. 
Definition 1.4. A subset $A$ of $M$ is said to be subanalytic if and only if, for every $x \in M$, there exist a neighborhood $U$ of $x$ in $M$ and $2 p$ couples $\left(\Phi_{i}^{\delta}, A_{i}^{\delta}\right)(1 \leqslant i \leqslant p$ and $\delta=1,2)$, where $A_{i}^{\delta} \in \operatorname{SEM}\left(M_{i}^{\delta}\right)$, and where the mappings $\Phi_{i}^{\delta}: M_{i}^{\delta} \rightarrow M$ are proper analytic, for real analytic manifolds $M_{i}^{\delta}$, such that

$$
A \cap U=\bigcup_{i=1}^{p}\left(\Phi_{i}^{1}\left(A_{i}^{1}\right) \backslash \Phi_{i}^{2}\left(A_{i}^{2}\right)\right) .
$$

Let $\operatorname{SUB}(M)$ denote the set of subanalytic subsets of $M$.

The subanalytic class is closed by union, intersection, complementary, inverse image by an analytic mapping, image by a proper analytic mapping. In brief, the subanalytic class is o-minimal (see [36]). Moreover subanalytic sets are stratifiable in the following sense.

Definition 1.5. Let $M$ be a differentiable manifold. A stratum in $M$ is a locally closed sub-manifold of $M$. A locally finite partition $\mathcal{S}$ of $M$ is a stratification of $M$ if any $S \in \mathcal{S}$ is a stratum such that

$$
\forall T \in \mathcal{S} \quad T \cap \operatorname{Fr} S \neq \emptyset \Rightarrow T \subset \operatorname{Fr} S \text { and } \operatorname{dim} T<\operatorname{dim} S .
$$

Finally, a mapping $f: M \rightarrow N$ between two analytic manifolds is said to be subanalytic if its graph is a subanalytic subset of $M \times N$.

Let $M$ be an analytic manifold, and $f$ be a subanalytic function on $M$. The analytic singular support of $f$, denoted $\mathcal{S}(f)$, is defined as the complement of the set of points $x$ in $M$ such that the restriction of $f$ to some neighborhood of $x$ is analytic. The following property is of great interest (see [32]).

Proposition 1.1. Let $f$ be a subanalytic function on an analytic manifold $M$. Then, its analytic singular support $\mathcal{S}(f)$ is subanalytic (and thus, in particular, is stratifiable). If $f$ is moreover locally bounded on $M$, then $\mathcal{S}(f)$ is of codimension greater than or equal to one.

A basic property of subanalytic functions, which makes them very useful in calculus of variations, and more generally in optimal control theory, is the following (see [32]).

Proposition 1.2. Let $M$ and $N$ be real analytic finite dimensional manifolds, $A$ be a subset of $N$, and $\Phi: N \rightarrow M$ and $f: N \rightarrow \mathbb{R}$ be subanalytic mappings. Define, for every $x \in M$,

$$
\psi(x)=\inf \left\{f(y) \mid y \in \Phi^{-1}(x) \cap A\right\} .
$$

If $\Phi_{\mid \bar{A}}$ is proper then $\psi$ is subanalytic.

This crucial tool in order to establish subanalyticity, based on an infimum property of the solution, suggests to investigate Hamilton-Jacobi equations stemming from optimization problems.

\subsection{Consequences on the singularities of viscosity solutions of Hamilton-Jacobi equations}

The paper will be organized as follows.

First, in the framework of the classical calculus of variations, where the Hamiltonian function is associated to an analytic Lagrangian function, we state existence, and in some cases, uniqueness of a subanalytic viscosity solution. These results specialize those of Lions [27] in the analytic case.

We then extend these statements to cases where the Hamiltonian function is stemming from sub-Riemannian geometry, and more generally from an optimal control problem, and show how the subanalyticity status of solutions is related to the existence of singular minimizing trajectories of the underlying control problem.

The results of this paper give conditions under which the viscosity solution of some Hamilton-Jacobi equations is subanalytic. Then, using Proposition 1.1, these results imply that the cut-locus, which coincides with the analytic singular set of the viscosity solution, is a subanalytic stratified manifold of codimension greater than or equal to one. 
Note that this singular set is also the set where characteristic curves intersect. The structural properties of this set have been much studied, and a usual way of investigating is to use nonsmooth analysis, and in particular semiconcave functions (see $[5,6,8,14,16,17,30,31]$ ). Here, we prove that, for some classes of Hamilton-Jacobi equations, under suitable assumptions, the viscosity solution is subanalytic and hence enjoys nice properties. In particular its singularities lie on a subanalytic stratified manifold of codimension greater than or equal to one.

This property is very useful in numerical analysis and has already been used (see [7]). The interest is to get a general framework in which the set where characteristic curves intersect is, in a sense, "small". Usual methods to derive such a fact rely on a careful analysis of the characteristic curves, that may be very involved. In the work [7], the stratification property of the singular set of the viscosity solution is essential in order to integrate energy functions on the set of characteristic curves.

The results of this paper, together with Proposition 1.1, provide systematic sufficient conditions under which this singular set shares these nice properties resulting from subanalyticity.

Some of the results of this article were announced in [35].

\section{Hamilton-Jacobi equations and calculus of variations}

In this section we specialize results of [27] in an analytic framework.

\subsection{A brief insight into calculus of variations}

We first recall the classical framework of calculus of variations. Let $H(x, p)$ be a $C^{2}$ function on $\mathbb{R}^{n} \times \mathbb{R}^{n}$, called Hamiltonian, satisfying the following assumptions:

- $H$ is uniformly superlinear, i.e.

$$
\forall A>0 \exists C(A) \in \mathbb{R} \mid \forall(x, p) \in \mathbb{R}^{n} \times \mathbb{R}^{n} \quad H(x, p) \geqslant A\|p\|-C(A),
$$

- $H$ is strictly convex in $p$, i.e. for all $(x, p) \in \mathbb{R}^{n} \times \mathbb{R}^{n}$ the second derivative

$$
\frac{\partial^{2} H}{\partial p^{2}}(x, p)
$$

is positive definite.

For all $(x, u) \in \mathbb{R}^{n} \times \mathbb{R}^{n}$, set

$$
L(x, u)=\sup _{p \in \mathbb{R}^{n}}(\langle p, u\rangle-H(x, p)) .
$$

It is well known that under the previous assumptions on $H$, this function, called the Lagrangian, is well defined on $\mathbb{R}^{n} \times \mathbb{R}^{n}$, and moreover satisfies also assumptions (2), (3), see for instance [22]. Moreover the so-called Legendre transformation

$$
\mathcal{T}(x, u)=\left(x, \frac{\partial L}{\partial u}(x, u)\right)
$$

is a global $C^{1}$ diffeomorphism on $\mathbb{R}^{n} \times \mathbb{R}^{n}$.

Definition 2.1. Let $\mathcal{A C}$ denote the set of absolutely continuous curves in $\mathbb{R}^{n}$.

- Let $x(\cdot) \in \mathcal{A C}$ be defined on $[0, T]$. The action of $x(\cdot)$ on $[0, T]$ is defined by

$$
\mathcal{A}_{T}(x(\cdot))=\int_{0}^{T} L(x(t), \dot{x}(t)) \mathrm{d} t .
$$

- For all $x, y \in \mathbb{R}^{n}$ define the value function at $(x, y)$ by

$$
S(x, y)=\inf \left\{\mathcal{A}_{T}(x(\cdot)) \mid T>0, x(\cdot) \in \mathcal{A C}, x(0)=y, x(T)=x\right\} .
$$


Assumption (3) actually implies that, for all $x, y \in \mathbb{R}^{n}$, there exists an absolutely continuous curve joining $y$ to $x$ and minimizing the action. This result is known as Tonelli Theorem (see [22]). In particular the infimum (7) is a minimum.

On the other part, minimizing curves are solutions of Euler-Lagrange equations

$$
\frac{\mathrm{d}}{\mathrm{d} t} \frac{\partial L}{\partial u}(x(t), \dot{x}(t))=\frac{\partial L}{\partial x}(x(t), \dot{x}(t)),
$$

which are equivalent, using the Legendre mapping, to the Hamilton equations

$$
\dot{x}(t)=\frac{\partial H}{\partial p}(x(t), p(t)), \quad \dot{p}(t)=-\frac{\partial H}{\partial x}(x(t), p(t)),
$$

where $p(t)$, called adjoint vector, is defined by

$$
(x(t), p(t))=\mathcal{T}(x(t), \dot{x}(t)) .
$$

Moreover, since the final time is not fixed, the identity

$$
H(x(t), p(t))=0
$$

holds along the trajectory.

A curve $x(\cdot) \in \mathcal{A C}$ for which there exists $p(\cdot) \in \mathcal{A C}$ such that Eqs. (8) and (9) hold is called an extremal.

Remark 2.1. If in the definition (11) of $S(x, y)$ the final time $T$ is fixed, then (9) does not hold.

\subsection{The Dirichlet problem}

The following theorem is an analytic version of [27, Theorem 5.3, p. 132].

Theorem 2.1. Let $\Omega$ denote a bounded subanalytic open subset of $\mathbb{R}^{n}$, c be a real number, and let $H(x, p)$ be an analytic Hamiltonian function on $\mathbb{R}^{n} \times \mathbb{R}^{n}$ satisfying assumptions (2), (3), and such that moreover

$$
\exists \alpha<c \mid \forall x \in \mathbb{R}^{n} \quad H(x, 0) \leqslant \alpha .
$$

Let $\mathcal{A C}$ denote the set of absolutely continuous curves in $\mathbb{R}^{n}$. For all $x, y \in \bar{\Omega}$, set

$$
S(x, y)=\inf \left\{\int_{0}^{T}(L(x(t), \dot{x}(t))+c) \mathrm{d} t \mid T>0, x(\cdot) \in \mathcal{A C}, x(0)=y, x(T)=x\right\},
$$

where $L$ is the Lagrangian associated to the Hamiltonian $H$. Then, for every $y_{0} \in \bar{\Omega}$, the function $x \mapsto S\left(x, y_{0}\right)($ resp., for every $x_{0} \in \bar{\Omega}$, the function $\left.y \mapsto S\left(x_{0}, y\right)\right)$ is a viscosity solution of

$$
H(x, \nabla v(x))-c=0 \quad \text { in } \Omega \backslash\left\{y_{0}\right\}, \quad v\left(y_{0}\right)=0,
$$

(resp., $H(x,-\nabla v(x))-c=0$ in $\Omega \backslash\left\{x_{0}\right\}, v\left(x_{0}\right)=0$ ), which is subanalytic on $\bar{\Omega}$.

Let $g$ denote a subanalytic function on $\Sigma=\partial \Omega$, satisfying the so-called compatibility condition

$$
\forall x, y \in \Sigma \quad g(x)-g(y) \leqslant S(x, y) .
$$

Finally, define, for every $x \in \bar{\Omega}$,

$$
S(x)=\inf _{y \in \Sigma}(g(y)+S(x, y)) .
$$

Then, $S$ is the unique viscosity solution of the Dirichlet problem

$$
\begin{aligned}
& H(x, \nabla v(x))-c=0 \quad \text { in } \Omega, \\
& v_{\mid \Sigma}=g,
\end{aligned}
$$

and moreover is continuous and subanalytic on $\bar{\Omega}$. 
Remark 2.2. If the Hamiltonian $H$ is defined on $\Omega \times \mathbb{R}^{n}$ and moreover assumptions (2), (3) and (10) hold only for every $x \in \Omega$, then the conclusion on $S(x)$ still holds. However, the definition of $S(x, y)$ has to be modified: the path joining $y$ to $x$ should be included in $\bar{\Omega}$, as done in [27] (see in particular [27, Remark 5.4, p. 118]. Moreover, in this case, we cannot prove the subanalyticity of the mappings $x \mapsto S\left(x, y_{0}\right)$ (resp., $y \mapsto S\left(x_{0}, y\right)$ ).

Remark 2.3. As noticed in [27, Remark 5.8, p. 128], this result also holds on an unbounded open set $\Omega$, except for uniqueness.

Example 2.1. Let $\Omega$ be a subanalytic bounded open set in $\mathbb{R}^{n}, \Sigma=\partial \Omega$, let $m(\cdot): \mathbb{R}^{n} \rightarrow \mathbb{R}^{n}$ be an analytic mapping, and $f(\cdot)$ be an analytic function on $\mathbb{R}^{n}$. Assume that there exists a positive real number $\varepsilon$ such that

$$
\|m(x)\|^{2} \leqslant f(x)^{2}-\varepsilon
$$

for every $x \in \mathbb{R}^{n}$. Then, there exists an unique viscosity solution $S$ of the Dirichlet problem

$$
\begin{aligned}
& \|\nabla v(x)-m(x)\|-|f(x)|=0 \quad \text { in } \Omega, \\
& v_{\mid \Sigma}=0,
\end{aligned}
$$

which is continuous and subanalytic on $\bar{\Omega}$.

This is indeed an easy consequence of Theorem 2.1, with the Lagrangian function

$$
L(x, u)=\frac{1}{2}\|u\|^{2}+\langle m(x), u\rangle+\frac{1}{2} f(x)^{2},
$$

for all $x, u \in \mathbb{R}^{n}$.

Proof of Theorem 2.1. For all $x \in \mathbb{R}^{n}$ and $u \in \mathbb{R}^{n}$, the assumption (10) implies the inequality $L(x, u)+c>0$, and thus $S(x, y)$ is well defined, for all $x, y \in \bar{\Omega}$.

Let $y_{0} \in \bar{\Omega}$ be fixed. It is proved in [27] that the function $x \mapsto S\left(x, y_{0}\right)$ is a viscosity solution of (12). We next prove that it is moreover subanalytic. To this aim, we express $S\left(x, y_{0}\right)$ using extremal curves. Absolutely continuous curves on $[0, T]$ such that $x(0)=y_{0}$, that are minimizing the action, are extremals, namely are solutions of (8). Hence, they are parametrized by $T$ and $p(0)$, where $p(\cdot)$ is an adjoint vector associated to $x(\cdot)$. This suggests to introduce the mapping

$$
\begin{aligned}
\Phi: \mathbb{R}^{+} \times \bar{\Omega} \times \mathbb{R}^{n} & \longrightarrow \mathbb{R}^{n}, \\
(T, y, \psi) & \longmapsto x(T)
\end{aligned}
$$

where $(x(\cdot), p(\cdot))$ is the solution of Eqs. (8) such that $x(0)=y$ and $p(0)=\psi$. The system (8) is analytic and hence $\Phi$ is an analytic mapping. We also set, with the same notations, for all $T \in \mathbb{R}^{+}, y \in \bar{\Omega}, \psi \in \mathbb{R}^{n}$,

$$
\sigma(T, y, \psi)=\int_{0}^{T}(L(x(t), \dot{x}(t))+c) \mathrm{d} t=\int_{0}^{T}\left(L\left(\mathcal{T}^{-1}(x(t), p(t))\right)+c\right) \mathrm{d} t .
$$

The Legendre mapping $\mathcal{T}$ is analytic, and thus $\sigma$ is also analytic. With these notations, we have

$$
S\left(x, y_{0}\right)=\inf \left\{\sigma\left(T, y_{0}, \psi\right) \mid \exists T \geqslant 0, \exists \psi \in \mathbb{R}^{n}, \Phi\left(T, y_{0}, \psi\right)=x\right\} .
$$

In order to apply Proposition 1.2, we have to prove that the couples $(T, \psi)$ in the last formula can be chosen in a compact subset of $\mathbb{R}^{n} \times \mathbb{R}^{n}$ as $x \in \bar{\Omega}$. By definition of the minimal action (11), the condition (9) leads to

$$
H\left(y_{0}, \psi\right)=c .
$$

Using the assumption (2), we infer that $\psi$ belongs to a compact set of $\mathbb{R}^{n}$ as $x \in \bar{\Omega}$. Moreover, the assumption (10) yields

$$
\sigma\left(T, y_{0}, \psi\right) \geqslant(c-\alpha) T,
$$


and since $\alpha<c$, we also get that $T$ is bounded. Finally, there exists a compact subset $K \subset \mathbb{R}^{+} \times \mathbb{R}^{n}$ such that

$$
S\left(x, y_{0}\right)=\inf \left\{\sigma\left(T, y_{0}, \psi\right) \mid \exists(T, \psi) \in K, \Phi\left(T, y_{0}, \psi\right)=x\right\} .
$$

Proposition 1.2 now asserts that the function $x \mapsto S\left(x, y_{0}\right)$ is subanalytic on $\bar{\Omega}$.

Finally, let $S$, the value function, be defined as in (14). It is continuous on $\bar{\Omega}$ because $S(x, y)$ is continuous. From [27], $S$ is a viscosity solution of (15). Uniqueness comes from assumption (10) and [10, Theorem 2.7, p. 37]. Subanalyticity of $S$ on $\bar{\Omega}$ is a consequence of Proposition 1.2.

\subsection{The Cauchy problem}

The following theorem is an analytic version of [27, Theorem 11.1, p. 217].

Theorem 2.2. Let $T>0$ be fixed, let $\Omega$ denote a bounded subanalytic open subset of $\mathbb{R}^{n}$, and $H(x, p)$ be an analytic Hamiltonian function on $\mathbb{R}^{n} \times \mathbb{R}^{n}$ satisfying assumptions (2), (3). For all $s, t \in[0, T]$ such that $s<t$, and all $x, y \in \bar{\Omega}$, set

$$
S(t, x, s, y)=\inf \left\{\int_{s}^{t} L(x(t), \dot{x}(t)) \mathrm{d} t \mid x(\cdot) \in \mathcal{A C}, x(s)=y, x(t)=x\right\} .
$$

Then, for all $s_{0} \in\left[0, T\left[\right.\right.$ and $y_{0} \in \bar{\Omega}$, the function $S\left(\cdot, \cdot, s_{0}, y_{0}\right)$ is a viscosity solution of

$$
\begin{aligned}
& \left.\left.\frac{\partial v}{\partial t}+H\left(x, \frac{\partial v}{\partial x}\right)=0 \quad \text { in }\right] s_{0}, T\right] \times \Omega \backslash\left\{y_{0}\right\}, \\
& \lim _{t \rightarrow s_{0}} v\left(t, y_{0}\right)=0,
\end{aligned}
$$

which is subanalytic on $\left.] s_{0}, T\right] \times \bar{\Omega}$. Let $g$ be a subanalytic function on $(\{0\} \times \bar{\Omega}) \cup([0, T] \times \partial \Omega)$ satisfying the compatibility condition

$$
\forall(s, y) \in(\{0\} \times \bar{\Omega}) \cup([0, T[\times \partial \Omega) \quad \forall(t, x) \in] s, T] \times \partial \Omega \quad g(t, x)-g(s, y) \leqslant S(t, x, s, y) .
$$

For all $t \in] 0, T]$ and $x \in \bar{\Omega}$, set

$$
S(t, x)=\inf \{S(t, x, s, y)+g(s, y) \mid(s, y) \in(\{0\} \times \bar{\Omega}) \cup([0, t[\times \partial \Omega)\} .
$$

Then, $S$ is continuous on $] 0, T] \times \bar{\Omega}$, is the unique viscosity solution of the Cauchy-Dirichlet problem

$$
\begin{aligned}
& \left.\frac{\partial v}{\partial t}+H\left(x, \frac{\partial v}{\partial x}\right)=0 \quad \text { in }\right] 0, T[\times \Omega, \\
& v=g \quad \text { on }] 0, T] \times \partial \Omega, \\
& \lim _{t \rightarrow 0} v(t, x)=g(0, x),
\end{aligned}
$$

and moreover $S$ is subanalytic on $] 0, T] \times \bar{\Omega}$.

Proof. The proof is similar to the proof of Theorem 2.1, except for the compactness argument needed to apply Proposition 1.2. We have with similar notations

$$
S\left(t, x, s_{0}, y_{0}\right)=\inf \left\{\sigma\left(t, s_{0}, y_{0}, \psi\right) \mid \Phi\left(t, s_{0}, y_{0}, \psi\right)=x\right\},
$$

where $\sigma$ and $\Phi$ are analytic mappings. Let us prove that $S\left(\cdot, \cdot, s_{0}, y_{0}\right)$ is subanalytic on any compact subset $I \times K$ of ]$\left.s_{0}, T\right] \times \bar{\Omega}$. First of all, notice that extremals are $C^{1}$ functions of $t$, for the Legendre mapping is $C^{1}$. In particular the function $t \mapsto L(x(t), \dot{x}(t))$ is $C^{1}$ along an extremal curve.

The following argument can for instance be found in [22]. By a continuity argument we can assert that the set

$$
\left\{\int_{s_{0}}^{t} L(x(t), \dot{x}(t)) \mathrm{d} t \mid(t, x(t)) \in I \times K, x(\cdot) \text { extremal, } x\left(s_{0}\right)=y_{0}\right\}
$$


is bounded. On the other part, for all extremal curve $x(\cdot)$, there exists from the Mean Value Theorem a real number $t_{0} \in[0, T]$ such that

$$
\int_{s_{0}}^{t} L(x(t), \dot{x}(t)) \mathrm{d} t=\left(t-s_{0}\right) L\left(x\left(t_{0}\right), \dot{x}\left(t_{0}\right)\right) .
$$

By continuity of the extremal flow, we infer easily that the set

$$
\left\{\dot{x}(0) \mid x(\cdot) \text { extremal, } x\left(s_{0}\right)=y_{0}, x(t)=x,(t, x) \in I \times K\right\}
$$

is bounded in $\mathbb{R}^{n}$ (see [22, Chapter 4] for all details). Therefore, the same holds for the corresponding initial adjoint vectors $p(0)$. The conclusion is then similar to the proof of Theorem 2.1.

\section{Hamilton-Jacobi equations and optimal control}

\subsection{A brief insight into optimal control theory}

Consider a general control system in $\mathbb{R}^{n}$

$$
\dot{x}_{u}(t)=f\left(x_{u}(t), u(t)\right),
$$

where $f: \mathbb{R}^{n} \times \mathbb{R}^{m} \rightarrow \mathbb{R}^{n}$ is smooth, and the controls $u$ belong to an open subset of $L_{\text {loc }}^{\infty}\left(\mathbb{R}, \mathbb{R}^{m}\right)$. For every $T>0$, denote by $\mathcal{U}_{T}$ the set of admissible controls on $[0, T]$, i.e. the set of controls such that the associated trajectory $x_{u}(\cdot)$ is well defined on $[0, T]$. It is an open subset of $L^{\infty}\left([0, T], \mathbb{R}^{m}\right)$. For every $x_{0} \in \mathbb{R}^{n}$, define on $\mathcal{U}_{T}$ the end-point mapping $E_{x_{0}, T}: u \mapsto x_{u}(T)$, where $x_{u}(\cdot)$ denotes the solution of (22) associated to the control $u \in \mathcal{U}_{T}$ and starting from $x_{0}$ at time $t=0$. It is a smooth mapping. A trajectory $x_{u}(\cdot)$ is said to be singular on $[0, T]$ if $u$ is a singular point of the end-point mapping $E_{x_{0}, T}$.

Let $M_{1}$ be a submanifold of $\mathbb{R}^{n}$. Consider the optimal control problem of determining, among all trajectories solutions of system (22) joining $x_{0}$ to $M_{1}$, a trajectory minimizing the cost function

$$
C(t, u)=\int_{0}^{t} f^{0}\left(x_{u}(s), u(s)\right) \mathrm{d} s+g\left(x_{u}(t)\right),
$$

where $f^{0}: \mathbb{R}^{n} \times \mathbb{R}^{m} \rightarrow \mathbb{R}$ and $g: \mathbb{R}^{n} \rightarrow \mathbb{R}$ are smooth functions, and $x_{u}(t) \in M_{1}$. Set moreover

$$
C_{0}(t, u)=\int_{0}^{t} f^{0}\left(x_{u}(s), u(s)\right) \mathrm{d} s .
$$

If a control $u$, associated to a trajectory $x_{u}(\cdot)$, is optimal on $[0, T]$, then there exists a nontrivial Lagrange multiplier $\left(\psi, \psi^{0}\right) \in \mathbb{R}^{n} \times \mathbb{R}$ such that

$$
\psi \cdot \mathrm{d} E_{x_{0}, T}(u)=-\psi^{0} \frac{\partial C_{0}}{\partial u}(T, u) .
$$

Moreover, $\psi-\psi^{0} \nabla g\left(x_{u}(T)\right) \perp T_{x_{u}(T)} M_{1}$. This is a first-order necessary condition for optimality. The well known Pontryagin Maximum Principle (see [28]) parametrizes this condition, and asserts that the trajectory $x_{u}(\cdot)$ corresponding to this control is the projection of an extremal, that is a solution of the Hamiltonian system

$$
\dot{x}_{u}=\frac{\partial H}{\partial p}\left(x_{u}, p_{u}, p_{u}^{0}, u\right), \quad \dot{p}_{u}=-\frac{\partial H}{\partial x}\left(x_{u}, p_{u}, p_{u}^{0}, u\right), \quad \frac{\partial H}{\partial u}\left(x_{u}, p_{u}, p_{u}^{0}, u\right)=0,
$$

where $H\left(x, p, p^{0}, u\right)=\langle p, f(x, u)\rangle+p^{0} f^{0}(x, u)$ is the Hamiltonian of the system, $p_{u}(\cdot):[0, T] \rightarrow \mathbb{R}^{n}$ is an absolutely continuous mapping called adjoint vector, and $p_{u}^{0}$ is a real nonpositive number. Moreover there holds at the final time, up to a multiplying scalar

$$
\left(p_{u}(T), p_{u}^{0}\right)=\left(\psi, \psi^{0}\right) .
$$

If $p_{u}^{0} \neq 0$, the extremal is said to be normal, and in this case it is normalized to $p_{u}^{0}=-1 / 2$. If $p_{u}^{0}=0$, the extremal is said to be abnormal. 
Remark 3.1. Since we did not set any constraint on the control, any singular trajectory is the projection of an abnormal extremal, and conversely.

Remark 3.2. As a consequence of the Maximum Principle, if a control $u$ is singular on $[0, T]$ then it is singular on $[0, t]$, for every $t \in] 0, T]$.

\subsection{Affine Dirichlet problem}

\subsubsection{The main results}

It is known that the existence of singular minimizing trajectories is closely related to the subanalyticity of the value function associated to an optimal control problem (see $[1,3,33,34]$ ). In these conditions, the following result is not surprising.

Theorem 3.1. Let $\Omega$ be a bounded subanalytic open subset of $\mathbb{R}^{n}, c>0$ be fixed, and $f_{0}, \ldots, f_{m}$ be analytic vector fields on $\bar{\Omega}$. For all $x \in \Omega$ and $p \in \mathbb{R}^{n}$, set

$$
H(x, p)=-\left\langle p, f_{0}(x)\right\rangle+\frac{1}{4} \sum_{i=1}^{m}\left\langle p, f_{i}(x)\right\rangle^{2}-c .
$$

Let $\Sigma=\partial \Omega$ and $g$ be a subanalytic function on $\Sigma$. For every $x \in \bar{\Omega}$, consider the optimal control problem of steering $x$ to $\Sigma$ for the affine control system

$$
\dot{x}_{u}(t)=f_{0}\left(x_{u}(t)\right)+\sum_{i=1}^{m} u_{i}(t) f_{i}\left(x_{u}(t)\right)
$$

and the cost

$$
C(u)=\int_{0}^{t(x, u)}\left(\sum_{i=1}^{m} u_{i}(s)^{2}+c\right) \mathrm{d} s+g\left(x_{u}(t(x, u))\right),
$$

where $t(x, u)$ is the infimum of times $t$ such that the solution $x_{u}(\cdot)$ of the control system (24) associated to the control $u$ steers the point $x \in \bar{\Omega}$ to $\Sigma$ in time $t$. We make the following assumptions.

- The boundary $\Sigma$ is accessible from $\Omega$, i.e., for every $x \in \Omega$, there exists a time $t$ and a control on $[0, t]$ such that the solution of the system (24) associated to this control and starting fromx at time 0 , joins $\Sigma$ in time $t$.

- There exists no singular minimizing trajectory of the control system (24) for the cost (25), steering $\Omega$ to $\Sigma$.

Let $S$ denote the value function associated to the optimal control problem (24), (25). Namely, if $\mathcal{S}$ denotes the set of solutions $(u(\cdot), x(\cdot))$ of $(24)$ defined on various intervals $\left[0, t_{1}\right]$, such that $x(0) \in \bar{\Omega}$ and $x\left(t_{1}\right) \in \Sigma$, one has, for every $x \in \bar{\Omega}$,

$$
S(x)=\inf \left\{C(u) \mid\left(u(\cdot), x_{u}(\cdot)\right) \in \mathcal{S}, x_{u}(0)=x\right\} .
$$

For all $x, z \in \Sigma$, define

$$
L(x, z)=\inf \left\{\int_{0}^{t}\left(\sum_{i=1}^{m} u_{i}(s)^{2}+c\right) \mathrm{d} s \mid x_{u}(\cdot) \in \mathcal{S}, x_{u}(0)=x, x_{u}(t)=z, \text { and } x_{u}(s) \in \bar{\Omega}, \forall s \in[0, t]\right\},
$$

and assume that $g$ satisfies the compatibility condition

$$
\forall x, z \in \Sigma \quad g(x)-g(z) \leqslant L(x, z) .
$$


Then $S$ is well defined on $\bar{\Omega}$, is continuous and subanalytic on $\Omega$, and is a viscosity solution of the Dirichlet problem

$$
H(x, \nabla S(x))=0 \quad \text { on } \Omega, \quad S_{\mid \Sigma}=g .
$$

Remark 3.3. Denote by $\mathcal{F}_{m}$ the set of $(m+1)$-uples of linearly independent vector fields $\left(f_{0}, \ldots, f_{m}\right)$, endowed with the $C^{\infty}$ Whitney topology. If $2 \leqslant m<n$, there exists an open dense subset of $\mathcal{F}_{m}$ such that every affine control system associated to a $(m+1)$-uple of this subset admits no singular minimizing trajectory. This is indeed an obvious extension of a result of [19] (see also [18, Corollary 3.4]).

Remark 3.4. If there exist singular minimizing trajectories, then the conclusion on subanalyticity of $S$ may fail (see Section 3.2.3).

We can state further results concerning uniqueness and regularity of $S$ on the whole $\bar{\Omega}$.

Proposition 3.2. Under the assumptions of Theorem 3.1, if moreover

$$
\forall x \in \Sigma \quad \operatorname{Lie}\left(f_{1}(x), \ldots, f_{m}(x)\right)=\mathbb{R}^{n},
$$

then $S$ is continuous on $\bar{\Omega}$. As a consequence, $S$ is the unique viscosity solution of the Dirichlet problem (31).

For every $x \in \mathbb{R}^{n}$, set

$$
\begin{aligned}
& \Delta(x)=\operatorname{Span}\left\{f_{1}(x), \ldots, f_{m}(x)\right\}, \\
& \Delta^{2}(x)=\Delta(x)+\operatorname{Span}\left\{\left[f_{i}, f_{j}\right](x), 1 \leqslant i<j \leqslant m\right\} .
\end{aligned}
$$

The $m$-uple $\left(f_{1}, \ldots, f_{m}\right)$ is said to be medium-fat at $x$ if, for every vector field $X \in \Delta(x) \backslash\{0\}$, there holds

$$
\mathbb{R}^{n}=\Delta^{2}(x)+\operatorname{Span}\left\{\left[X,\left[f_{i}, f_{j}\right]\right](x), 1 \leqslant i, j \leqslant m\right\} .
$$

Proposition 3.3. Under the assumptions of Theorem 3.1, if the m-uple of vector fields $\left(f_{1}, \ldots, f_{m}\right)$ is moreover medium-fat at every point of $\Sigma$, and if the compatibility inequality (30) is strict, then $S$ is subanalytic on $\bar{\Omega}$.

Remark 3.5. If $n \leqslant m(m-1)+1$, then the $m$-uple of vector fields $\left(f_{1}, \ldots, f_{m}\right)$ is generically (in $C^{\infty}$ Whitney topology) medium-fat (see [4]).

The proofs of Theorem 3.1, Propositions 3.2 and 3.3 are provided, resp., in Sections 3.2.4, 3.2.5 and 3.2.6.

Remark 3.6. Assumption (32) actually implies that the affine control system (24) is STC $\Sigma$, i.e. small-time controllable near $\Sigma$ (see [9] for a definition). For a proof of this fact, we refer the reader for instance to [12,26]. In [9] where the controls take their values in a compact subset of $\mathbb{R}^{m}$, this small-time controllability property is used to prove that $S$ is upper semi-continuous. Their proof is however not adapted in our framework since our controls are not constrained (see also Remark 3.19).

On the other part, the compatibility condition of [9] is stronger than (30). Coupled with small-time controllability, it enables to prove that the value function $S$ is continuous. Here the continuity of $S$ is mainly due to the assumption (27) of the absence of singular minimizers.

Example 3.1. Let $\Omega$ be a subanalytic bounded open subset of $\mathbb{R}^{3}$ and $\Sigma=\partial \Omega$. For every $c>0$, and every $\alpha \in \mathbb{R}$, there exists a unique viscosity solution $S$ of the Dirichlet problem

$$
-\alpha \frac{\partial v}{\partial x_{1}}+\frac{1}{4}\left(\frac{\partial v}{\partial x_{1}}+x_{2} \frac{\partial v}{\partial x_{3}}\right)^{2}+\frac{1}{4}\left(\frac{\partial v}{\partial x_{2}}-x_{1} \frac{\partial v}{\partial x_{3}}\right)^{2}-c=0 \quad \text { in } \Omega, \quad v_{\mid \Sigma}=0,
$$

which is continuous and subanalytic on $\bar{\Omega}$. It is indeed an application of Theorem 3.1, Propositions 3.2 and 3.3, with the vector fields

$$
f_{0}=\alpha \frac{\partial}{\partial x_{1}}, \quad f_{1}=\frac{\partial}{\partial x_{1}}+x_{2} \frac{\partial}{\partial x_{3}}, \quad f_{2}=\frac{\partial}{\partial x_{2}}-x_{1} \frac{\partial}{\partial x_{3}} .
$$




\subsubsection{Particular case: $f_{0}=0$}

If $f_{0}=0$, then the statement of Theorem 3.1 can be interpreted in the framework of sub-Riemannian geometry. Theorem 3.4 hereafter is actually more precise than the one of Theorem 3.1. In particular, it is of interest to interpret the viscosity solution of the corresponding Hamilton-Jacobi equation as a sub-Riemannian distance. Note that, in sub-Riemannian geometry, many things are known about the regularity of the distance (see $[1,3])$.

We first recall a general definition of a sub-Riemannian distance, due to [11]. Let $M$ be a smooth $n$-dimensional manifold, $m$ an integer such that $1 \leqslant m \leqslant n$, and $f_{1}, \ldots, f_{m}$ be smooth vector fields on a manifold $M$. For all $x \in M$ and $v \in T_{x} M$, set

$$
g(x, v)=\inf \left\{u_{1}^{2}+\cdots+u_{m}^{2} \mid u_{1}, \ldots, u_{m} \in \mathbb{R}, u_{1} f_{1}(x)+\cdots+u_{m} f_{m}(x)=v\right\} .
$$

Then, $g(x, \cdot)$ is a positive definite quadratic form on the subspace of $T_{x} M$ spanned by $f_{1}(x), \ldots, f_{m}(x)$. Outside this subspace, we set $g(x, v)=+\infty$. The form $g$ is called the sub-Riemannian metric associated to the m-uple of vector fields $\left(f_{1}, \ldots, f_{m}\right)$.

Let $\mathcal{A C}([0,1], M)$ denote the set of absolutely continuous paths in $M$, defined on $[0,1]$. Define the length of $\gamma \in \mathcal{A C}([0,1], M)$ by $l(\gamma)=\int_{0}^{1} \sqrt{g(\gamma(t), \dot{\gamma}(t))} \mathrm{d} t$. Finally, the sub-Riemannian distance associated to the $m$-uple of vector fields $\left(f_{1}, \ldots, f_{m}\right)$, between two points $x_{0}, x_{1}$ in $M$, is defined by

$$
d_{S R}\left(x_{0}, x_{1}\right)=\inf \left\{l(\gamma) \mid \gamma \in \mathcal{A} C([0,1], M), \gamma(0)=x_{0}, \gamma(1)=x_{1}\right\} .
$$

Consider on the other part the differential system on $T M$

$$
\dot{x}(t)=\sum_{i=1}^{m} u_{i}(t) f_{i}(x(t)) \quad \text { a.e. on }[0,1],
$$

where the function $u(\cdot)=\left(u_{1}(\cdot), \ldots, u_{m}(\cdot)\right)$, called control function, belongs to $L^{2}\left([0,1], \mathbb{R}^{m}\right)$. Let $x_{0} \in \mathbb{R}^{n}$, and let $\mathcal{U}$ denote the (open) subset of $L^{2}\left([0,1], \mathbb{R}^{m}\right)$ such that every solution of (33), starting from $x_{0}$, and associated to a control $u \in \mathcal{U}$, is well defined on [0,1]. The mapping $E_{x_{0}}: u \in \mathcal{U} \mapsto x(1) \in \mathbb{R}^{n}$, which to a control $u$ associates the extremity $x(1)$ of the corresponding solution $x(\cdot)$ of (33) such that $x(0)=x_{0}$, is called end-point mapping at the point $x_{0}$. It is a smooth mapping. The trajectory $x(\cdot)$ is said to be singular if the associated control $u$ is a singular point of the end-point mapping. It is said minimizing if it realizes the sub-Riemannian distance between its extremities.

Remark 3.7. A sub-Riemannian manifold is often defined as a triple $(M, \Delta, g)$, where $M$ is a $n$-dimensional manifold, $\Delta$ is a distribution of rank $m$ (with $m \leqslant n$ ), and $g$ is a Riemannian metric on $\Delta$. Here, as in [11], and for the needs of applications, the point of view is more general and the assumption of constant rank is relaxed.

Notice that, if the vector fields $\left(f_{1}, \ldots, f_{m}\right)$ are everywhere linearly independent, then controlled paths solutions of (33) coincide with absolutely continuous paths tangent to the distribution $\Delta$, where

$$
\Delta(x)=\operatorname{Span}\left\{f_{1}(x), \ldots, f_{m}(x)\right\}
$$

for every $x \in M$. On the other part the set of absolutely continuous paths which are tangent to $\Delta$ is not in general a manifold: its singularities correspond exactly to singular trajectories of the control system (33), which are intrinsic to the distribution $\Delta$.

Theorem 3.4. Let $\Omega$ denote a bounded subanalytic open subset of $\mathbb{R}^{n}, m \geqslant 1$, be an integer, and let $H(x, p)$ be an Hamiltonian function on $\mathbb{R}^{n} \times \mathbb{R}^{n}$ defined by

$$
H(x, p)=\sum_{i=1}^{m}\left\langle p, f_{i}(x)\right\rangle^{2}
$$

where $f_{1}, \ldots, f_{m}$ are analytic vector fields on $\mathbb{R}^{n}$ satisfying the Hörmander condition

$$
\forall x \in \mathbb{R}^{n} \quad \operatorname{Lie}\left(f_{1}(x), \ldots, f_{m}(x)\right)=\mathbb{R}^{n},
$$

i.e. the Lie algebra spanned by the vector fields has maximal rank at every point. Let $d_{S R}(\cdot, \cdot)$ denote the subRiemannian distance associated to the $m$-uple of vector fields $\left(f_{1}, \ldots, f_{m}\right)$. 
Then, for every $y_{0} \in \mathbb{R}^{n}$, the function $x \mapsto d_{S R}\left(x, y_{0}\right)$ is a viscosity solution of

$$
H(x, \nabla v(x))-1=0 \quad \text { in } \mathbb{R}^{n} \backslash\left\{y_{0}\right\}, \quad v\left(y_{0}\right)=0 .
$$

Moreover, under the additional assumption that the control system (33) has no nontrivial singular minimizer starting from $y_{0}$, the function $d_{S R}\left(\cdot, y_{0}\right)$ is subanalytic on $\mathbb{R}^{n} \backslash\left\{y_{0}\right\}$.

Let $g$ denote a subanalytic function on $\Sigma=\partial \Omega$, satisfying the compatibility condition

$$
\forall x, y \in \Sigma \quad g(x)-g(y) \leqslant d_{S R}(x, y),
$$

and for every $x \in \bar{\Omega}$ set

$$
S(x)=\inf _{y \in \Sigma}\left(g(y)+d_{S R}(x, y)\right) .
$$

Then, $S$ is continuous on $\bar{\Omega}$ and is the unique viscosity solution of the Dirichlet problem

$$
H(x, \nabla v(x))-1=0 \quad \text { in } \Omega, \quad v_{\mid \Sigma}=g .
$$

If moreover the control system (33) has no nontrivial singular minimizer starting from $\Sigma$, then $S$ is subanalytic on $\Omega$.

Remark 3.8. The same comments as in Remarks 2.2 and 2.3 hold.

Remark 3.9. If $m \geqslant n$, we are in the Riemannian situation and there exists no singular trajectory.

Remark 3.10. Denote by $\mathcal{F}_{m}$ the set of $m$-uples of linearly independent vector fields $\left(f_{1}, \ldots, f_{m}\right)$, endowed with the $C^{\infty}$ Whitney topology. If $m \geqslant 3$, there exists an open dense subset of $\mathcal{F}_{m}$ such that any sub-Riemannian system associated to a $m$-uple of this subset admits no nontrivial singular minimizer, see [18,19] (see also [3] for the existence of a dense set only). Hence, generically, the mapping $S$ is subanalytic on $\Omega$, without assuming the absence of singular minimizers.

Remark 3.11. According to Proposition 3.3, under the assumptions of Theorem 3.4, if the $m$-uple of vector fields $\left(f_{1}, \ldots, f_{m}\right)$ is moreover medium-fat at every point of $\Sigma$, then $S$ is subanalytic on the whole $\bar{\Omega}$.

The proof of Theorem 3.4 can actually be derived from of Theorem 3.1. However, since it is easy to achieve directly, we next provide a short proof of this result.

Proof of Theorem 3.4. First of all, notice that the sub-Riemannian problem is in fact equivalent to the time-optimal problem for the control system

$$
\dot{x}(t)=\sum_{i=1}^{m} u_{i}(t) f_{i}(x(t)), \quad \sum_{i=1}^{m} u_{i}(t)^{2} \leqslant 1
$$

(see [11]). Moreover, the sub-Riemannian distance $d_{S R}(x, y)$ is equal to the minimal time $T(x, y)$ needed to join $x$ to $y$ for this system. On the other part, Hörmander's condition (35) implies that, for each couple $(x, y)$ of points of $\mathbb{R}^{n}$, there exists a minimizing curve joining $x$ to $y$, that is $d_{S R}(x, y)<+\infty$ (it is a consequence of Chow's theorem, see [11]).

Let $y_{0} \in \mathbb{R}^{n}$ be fixed. From [9, Proposition 2.3, p. 240] the function $x \mapsto T\left(x, y_{0}\right)$ is a viscosity solution of $H(x, \nabla u(x))-1=0$ on $\mathbb{R}^{n} \backslash\left\{y_{0}\right\}$. If moreover the sub-Riemannian system admits no nontrivial singular minimizer starting from $y_{0}$ then this function is subanalytic outside $y_{0}$ (see $[1,3,34]$ ).

Introduce $S$ as in (38), and notice that for all $x, y \in \bar{\Omega}$,

$$
\begin{aligned}
S(x)-S(y) & =\inf _{z \in \Sigma}\left(d_{S R}(x, z)+g(z)\right)-\inf _{z \in \Sigma}\left(d_{S R}(y, z)+g(z)\right) \\
& \leqslant \sup _{z \in \Sigma}\left(d_{S R}(x, z)-d_{S R}(x, y)\right) \\
& \leqslant d_{S R}(x, y),
\end{aligned}
$$




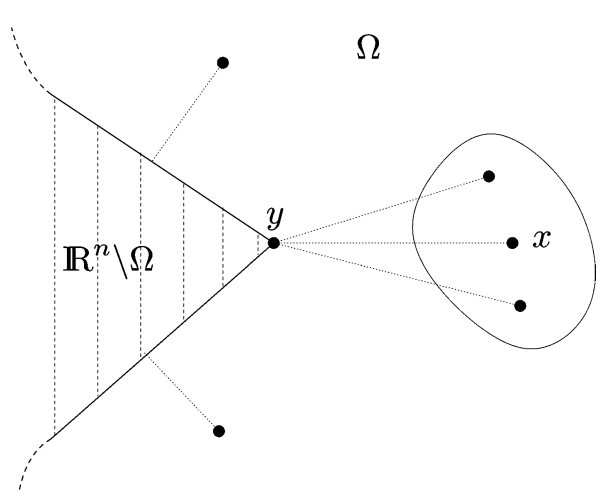

Fig. 1. Analogy with the Euclidean distance.

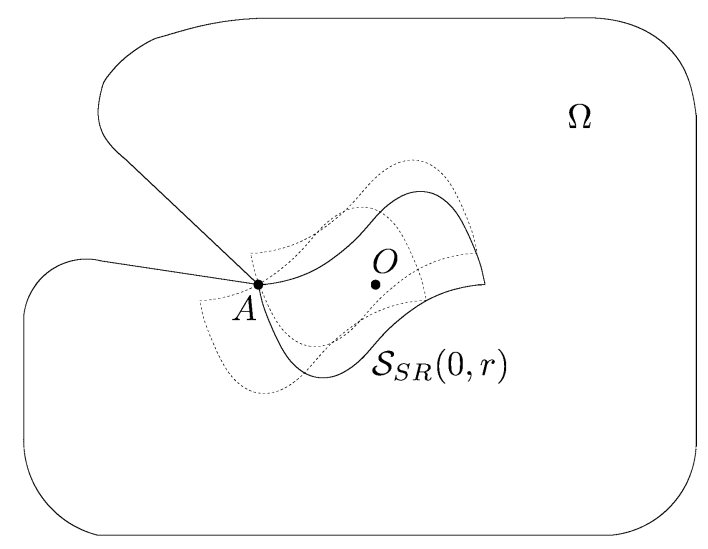

Fig. 2. The Martinet case.

and thus $S$ is continuous on $\bar{\Omega}$. The fact that $S$ is a viscosity solution of (39) is again a consequence of [9]. Under the compatibility condition (37) uniqueness comes from [10, Theorem 2.7, p. 37]. Finally, if there is no nontrivial singular minimizer, Proposition 1.2 implies the subanalyticity of $S$ on $\Omega$.

\subsubsection{A counterexample to subanalyticity}

The counterexample that we are going to construct is based on the so-called Martinet case in sub-Riemannian geometry. We briefly recall the context, see $[2,13,34]$. Consider in $\mathbb{R}^{3}$ the two vector fields

$$
f_{1}\left(x_{1}, x_{2}, x_{3}\right)=\frac{\partial}{\partial x_{1}}+x_{2}^{2} \frac{\partial}{\partial x_{3}}, \quad f_{2}\left(x_{1}, x_{2}, x_{3}\right)=\frac{\partial}{\partial x_{2}} .
$$

There are only two singular trajectories starting at time $t=0$ from the origin, namely $t \mapsto(t, 0,0)$ and $t \mapsto(-t, 0,0)$, which are moreover minimizing. The associated sub-Riemannian distance to the origin, namely the mapping $d_{S R}(0, \cdot)$, can be proved to be not subanalytic along the axis $\left(0 x_{1}\right)$, and moreover the shape of the sub-Riemannian spheres $\mathcal{S}_{S R}(0, r)$ near a point of this axis is well known (see Fig. 2).

Here, according to Theorem 3.4, we need rather consider a distance to a set. Let us explain the idea by analogy with the Euclidean distance to a set in the plane: it is quite clear in this case how to construct a corner-shaped bounded open set $\Omega$ such that there exist a point $y \in \partial \Omega$ (at the corner), a point $x \in \Omega$ and a neighborhood of $x$ in which the distance to $\partial \Omega$ is equal to the distance to the point $y$ (see Fig. 1).

Analogously, in the Martinet case, we claim that there exists an subanalytic open bounded set $\Omega \subset \mathbb{R}^{n}$ containing the origin in its interior, such that the function

$$
S(x)=\inf _{y \in \partial \Omega} d_{S R}(x, y)
$$

can be written, in some neighborhood of the origin, as

$$
S(x)=d_{S R}(x, A),
$$

where the point $A$ is defined as $A=(r, 0,0), r \neq 0$ (see Fig. 2).

From Theorem 3.4, $S$ is the unique viscosity solution of the Dirichlet problem

$$
\left(\frac{\partial v}{\partial x_{1}}+x_{2}^{2} \frac{\partial v}{\partial x_{3}}\right)^{2}+\left(\frac{\partial v}{\partial x_{2}}\right)^{2}-1=0 \quad \text { in } \Omega, \quad v_{\mid \partial \Omega}=0
$$

The corresponding Hamiltonian

$$
H(x, p)=\left(p_{1}+p_{3} x_{2}^{2}\right)^{2}+p_{2}^{2}
$$

is analytic on $\mathbb{R}^{n} \times \mathbb{R}^{n}$. Anyway, $S$ is not subanalytic in a neighborhood of the origin, on the axis $\left(0 x_{1}\right)$.

Remark 3.12. It is not difficult to construct explicitly the set $\Omega$, since the asymptotics of the sub-Riemannian spheres with small radius is known precisely (see [13]). 
In this example, all data are analytic and anyway the unique viscosity solution is not subanalytic, due to the existence of singular minimizing trajectories. This phenomenon is not exceptional and is, in a sense, generic, as explained in the next remark. In fact, the Martinet case can be imbedded into generic distributions (see [3]).

Remark 3.13. The function $S$ may happen not to be subanalytic on $\Sigma$. Indeed, a result from [3] asserts that for generic $m$-uples of vector fields $\left(f_{1}, \ldots, f_{m}\right)$ in $\mathbb{R}^{n}$, if $m \geqslant 3$ and $n \leqslant(m-1)\left(\frac{m^{2}}{3}+\frac{5 m}{6}+1\right)$, then the sub-Riemannian distance at 0 , namely the function $x \mapsto d_{S R}(0, x)$, is not subanalytic at 0 , whereas sub-Riemannian spheres $\mathcal{S}_{S R}(0, r)$ with small radius $r>0$ are subanalytic. For such data, let $B_{S R}(0, r)$ denote the sub-Riemannian ball centered at 0 and with radius $r$, and set $\Omega=B_{S R}(0, r) \backslash\{0\}$. In these conditions, the boundary $\Sigma=\{0\} \cup \mathcal{S}_{S R}(0, r)$ of $\Omega$ is subanalytic. For every $x \in \Omega$, define

$$
S(x)=\inf \left(d_{S R}(0, x), d_{S R}\left(x, \mathcal{S}_{S R}(0, r)\right)\right),
$$

and, for every $x \in \Sigma$, set $g(x)=0$. Then, $S$ is the unique viscosity solution of (39) for the Hamiltonian function corresponding to $\left(f_{1}, \ldots, f_{m}\right)$, and $S$ is not subanalytic at 0 .

\subsubsection{Proof of Theorem 3.1}

First of all, the assumption (26) implies that $S$ is well defined on $\bar{\Omega}$. Let us prove that the infimum in the definition (28) of $S$ is actually a minimum. This is a consequence of the following lemma on the existence of optimal controls.

Lemma 3.5. For every $x \in \bar{\Omega}$, there exists a control $u$ minimizing the cost (25), such that the associated trajectory $x_{u}(\cdot)$ satisfies $x_{u}(0)=x, x_{u}(t(x, u)) \in \Sigma$.

Proof of Lemma 3.5. If $x \in \Sigma$, the conclusion is immediate. If $x \in \Omega$, consider a sequence of controls $\left(u_{n}\right)_{n \in \mathbb{N}}$, where $u_{n} \in L^{2}\left(\left[0, t_{n}\right], \mathbb{R}^{m}\right)$, such that $C\left(u_{n}\right)$ converges towards the infimum $S(x)$. Here we denote $t_{n}=t\left(x, u_{n}\right)$. Assumption (26) implies that the sequence of real numbers $\left(C\left(u_{n}\right)\right)_{n \in \mathbb{N}}$ is bounded. Since $g$ is bounded on the compact set $\Sigma$ and $c>0$, one easily gets on the one part that the sequence of real numbers $\left(t_{n}\right)_{n \in \mathbb{N}}$ is bounded, and on the other part that the sequence $\left(u_{n}\right)_{n \in \mathbb{N}}$ is bounded in $L^{2}\left([0, T], \mathbb{R}^{m}\right)$, where $T$ is a real greater than each $t_{n}$, and where $u_{n}(\cdot)$ is extended by 0 on $\left.] t_{n}, T\right]$. Hence, up to a subsequence, we can assert that $t_{n}$ tends to a real number $t>0$ and $u_{n}$ tends to a control $u \in L^{2}\left([0, t], \mathbb{R}^{m}\right)$ in the weak $L^{2}$-topology. Since the control system (24) is affine, it is not difficult to prove that the sequence $\left(x_{u_{n}}(\cdot)\right)_{n \in \mathbb{N}}$ converges uniformly towards $x_{u}(\cdot)$ on $[0, t]$ (see [33] for details), where $x_{u}(\cdot)$ is the trajectory associated to the control $u$. In particular $x_{u}(s) \in \bar{\Omega}$ for all $s \in[0, t]$, and since $x_{u_{n}}\left(t_{n}\right) \in \Sigma$ we infer that $x_{u}(t) \in \Sigma$.

On the other part, using the weak convergence of $u_{n}$ towards $u$, one has

$$
\int_{0}^{t}\left(\sum_{i=1}^{m} u_{i}(s)^{2}+c\right) \mathrm{d} s+g\left(x_{u}(t)\right) \leqslant \liminf C\left(u_{n}\right)=S(x),
$$

and since $S(x)$ is an infimum, this inequality is actually an equality. Thus the control $u$ steers the system from $x$ to $\Sigma$ in time $t$, with a cost $S(x)$. By definition of $t(x, u)$, it is clear that $t(x, u) \leqslant t$, anyway equality does not hold necessarily. At this stage we need the compatibility assumption (30), which yields the inequality

$$
g\left(x_{u}(t(x, u))\right)-g\left(x_{u}(t)\right) \leqslant L\left(x_{u}(t(x, u)), x_{u}(t)\right) .
$$

Moreover, from the definition of $L$,

$$
L\left(x_{u}(t(x, u)), x_{u}(t)\right) \leqslant \int_{t(x, u)}^{t}\left(\sum_{i=1}^{m} u_{i}(s)^{2}+c\right) \mathrm{d} s,
$$

and hence

$$
\int_{0}^{t(x, u)}\left(\sum_{i=1}^{m} u_{i}(s)^{2}+c\right) \mathrm{d} s+g\left(x_{u}(t(x, u))\right) \leqslant \int_{0}^{t}\left(\sum_{i=1}^{m} u_{i}(s)^{2}+c\right) \mathrm{d} s+g\left(x_{u}(t)\right)=S(x),
$$


and as previously this inequality is actually an equality, i.e.

$$
S(x)=\int_{0}^{t(x, u)}\left(\sum_{i=1}^{m} u_{i}(s)^{2}+c\right) \mathrm{d} s+g\left(x_{u}(t(x, u))\right),
$$

which proves that the control $u$ is minimizing.

\section{Lemma 3.6. The function $S$ is continuous on $\Omega$.}

Proof of Lemma 3.6. Let $\left(x_{n}\right)_{n \in \mathbb{N}}$ be a sequence of points of $\Omega$ converging towards $x \in \Omega$. Let us prove that $S\left(x_{n}\right)$ tends to $S(x)$. We first show that the sequence $\left(S\left(x_{n}\right)\right)_{n \in \mathbb{N}}$ is bounded. From Lemma 3.5, there exists an optimal control $u$ steering the system from $x$ to $\Sigma$ in time $t(x, u)$, and from assumption (27) this control cannot be singular. Hence the end-point mapping $E_{x, t(x, u)}$ is a submersion at the point $u$, and consequently the equation

$$
E_{y, t(x, u)}(v)=x_{u}(t(x, u))
$$

may be solved in $v$ in a $L^{2}$-neighborhood of $u$, and for every $y \in \Omega$ close to $x$. In particular, if $n$ is large enough, then there exists a control $u_{n}$ close to $u$ in $L^{2}$-topology such that

$$
E_{x_{n}, t(x, u)}\left(u_{n}\right)=x_{u}(t(x, u)) .
$$

From the previous equality, $u_{n}$ is a control steering $x_{n}$ to $\Sigma$ in time $t(x, u)$, and since $u_{n}$ is close to $u$ in $L^{2}$-topology it is not difficult to infer that the sequence $\left(S\left(x_{n}\right)\right)_{n \in \mathbb{N}}$ is bounded.

Let $a$ be a cluster point of this sequence. To end the proof, we show that $S(x)=a$, i.e. $a$ is the unique cluster point of $\left(S\left(x_{n}\right)\right)_{n \in \mathbb{N}}$. First, we can assert that up to a subsequence $S\left(x_{n}\right)$ tends to $a$. From Lemma 3.5, for every integer $n$, there exists a minimizing control $u_{n}$ such that $S\left(x_{n}\right)=C\left(u_{n}\right)$. A reasoning similar to the proof of Lemma 3.5, using the compatibility condition (30), yields the inequality

$$
S(x) \leqslant a .
$$

Conversely, similarly as to prove that the sequence $\left(S\left(x_{n}\right)\right)_{n \in \mathbb{N}}$ is bounded, and using again (30), one states

$$
a=\liminf S\left(x_{n}\right) \leqslant C(u)=S(x),
$$

which ends the proof.

Lemma 3.7. The function $S$ is subanalytic on $\Omega$.

Proof of Lemma 3.7. Let us prove that $S$ is subanalytic on any subanalytic compact subset $K$ of $\Omega$. From Lemma 3.5, for every $x \in K$, there exists a minimizing control $u$ such that $S(x)=C(u)$, which cannot be singular by assumption. From the Pontryagin maximum principle, the associated trajectory $x_{u}(\cdot)$ is the projection of a normal extremal, i.e. there exists an adjoint vector $p_{u}(\cdot)$ on $[0, t(x, u)]$, each $p_{u}(t)$ being identified to a row vector, and a constant $p_{u}^{0}=$ $-1 / 2$ such that there holds

$$
\begin{aligned}
& \dot{x}_{u}(t)=f_{0}\left(x_{u}(t)\right)+\sum_{i=1}^{m} u_{i}(t) f_{i}\left(x_{u}(t)\right), \\
& \dot{p}_{u}(t)=-p_{u}(t) \frac{\partial f_{0}}{\partial x}\left(x_{u}(t)\right)-\sum_{i=1}^{m} u_{i}(t) p_{u}(t) \frac{\partial f_{i}}{\partial x}\left(x_{u}(t)\right),
\end{aligned}
$$

almost everywhere on $[0, t(x, u)]$, with $u_{i}(t)=p_{u}(t) f_{i}\left(x_{u}(t)\right)$. Hence, normal extremals are solutions of the differential system

$$
\dot{x}(t)=\frac{\partial H_{1}}{\partial p}(x(t), p(t)), \quad \dot{p}(t)=-\frac{\partial H_{1}}{\partial x}(x(t), p(t)),
$$


where

$$
H_{1}(x, p)=\left\langle p, f_{0}(x)\right\rangle+\frac{1}{2} \sum_{i=1}^{m}\left\langle p, f_{i}(x)\right\rangle^{2} .
$$

This is an analytic differential system in $(x, p)$ parametrized by the initial condition $p(0)=p_{0}$. In particular, normal extremals $(x(\cdot), p(\cdot))$ such that $x(0)=x_{0}$ depend analytically on the initial condition $p_{0}$. Notice moreover that the mapping

$$
\begin{aligned}
\Phi: \mathbb{R}^{n} \longrightarrow L^{2}\left([0, T], \mathbb{R}^{m}\right), \\
p_{0} \longmapsto u_{p_{0}},
\end{aligned}
$$

needed further, where $u_{p_{0}}$ denotes the corresponding normal control, is analytic.

On the other part, one has, at the final time $t(x, u)$, the Lagrange multipliers identity

$$
p_{u}(t(x, u)) \mathrm{d} E_{x, t(x, u)}(u)=\frac{1}{2} \frac{\partial C_{0}}{\partial u}(t(x, u), u),
$$

where

$$
C_{0}(t, u)=\int_{0}^{t}\left(\sum_{i=1}^{m} u_{i}(s)^{2}+c\right) \mathrm{d} s .
$$

It is clear that, for every control $u \in L^{2}\left([0, T], \mathbb{R}^{m}\right)$, the differential of $C_{0}$ with respect to $u$ writes

$$
\forall v \in L^{2}\left([0, T], \mathbb{R}^{m}\right) \quad \frac{\partial C_{0}}{\partial u}(T, u) \cdot v=2 \int_{0}^{T} \sum_{i=1}^{m} u_{i}(t) v_{i}(t) \mathrm{d} t .
$$

Hence, identifying $L^{2}$ with its dual space, Eq. (41) leads to the equality in $L^{2}\left([0, t(x, u)], \mathbb{R}^{m}\right)$

$$
p_{u}(t(x, u)) \mathrm{d} E_{x, t(x, u)}(u)=u .
$$

We next prove the following fact.

Fact 1. The set $\left\{p_{u}(t(x, u)) \mid x \in K\right.$ and $\left.S(x)=C(u)\right\}$ is compact in $\mathbb{R}^{n}$.

If not, there exists a sequence $\left(x_{n}\right)_{n \in \mathbb{N}}$ of points of $K$ converging towards $x \in K$, such that

$$
\left\|p_{u_{n}}\left(t_{n}\right)\right\| \rightarrow+\infty
$$

where $u_{n}$ is an optimal (necessarily nonsingular) control steering $x_{n}$ to $\Sigma$ in time $t_{n}=t\left(x_{n}, u_{n}\right)$. Since $S$ is continuous on $K$, the sequence $\left(S\left(x_{n}\right)\right)_{n \in \mathbb{N}}$ is bounded, and thus, up to a subsequence, we can assume that $t_{n}$ tends to a real $t$ and $u_{n}$ tends to $u \in L^{2}\left([0, t], \mathbb{R}^{m}\right)$ in weak $L^{2}$-topology. From (42), there holds

$$
p_{u_{n}}\left(t_{n}\right) \mathrm{d} E_{x_{n}, t_{n}}\left(u_{n}\right)=u_{n},
$$

for every integer $n$. Up to a subsequence we can assume that

$$
\frac{p_{u_{n}}\left(t_{n}\right)}{\left\|p_{u_{n}}\left(t_{n}\right)\right\|} \rightarrow \psi
$$

where $\psi \in \mathbb{R}^{n} \backslash\{0\}$. Taking the limit in (43), we infer

$$
\psi \mathrm{d} E_{x, t}(u)=0 .
$$

Indeed, for an affine control system, the end-point mapping and its differential can be easily proved to be continuous with respect to the weak $L^{2}$-topology (see [33] for a proof and for a similar reasoning). On the other part, since $x_{n}$ tends to $x$, we get

$$
E_{x, t}(u) \in \Sigma \text {. }
$$


As a consequence of (44) and (45), $u$ is a singular control on $[0, t]$ steering $x$ to $\Sigma$ in time $t$. It is still singular on $[0, t(x, u)]$; let us prove that it is optimal on this interval. Indeed, each control $u_{n}$ is optimal, i.e. $C\left(u_{n}\right)=S\left(x_{n}\right)$. Since $t_{n}$ tends to $t$ and $u_{n}$ tends to $u$ in weak $L^{2}$-topology, we get

$$
C_{0}(t, u)+g\left(x_{u}(t)\right) \leqslant \liminf S\left(x_{n}\right)=S(x),
$$

for $S$ is continuous. Moreover, using the compatibility condition (30) leads to

$$
C(u)=C_{0}(t(x, u), u)+g\left(x_{u}(t(x, u))\right) \leqslant C_{0}(t, u)+g\left(x_{u}(t)\right) \leqslant S(x),
$$

thus this inequality is actually an equality, i.e. $u$ is optimal. This contradiction with assumption (27) ends the proof of Fact 1 .

Fact 2. The set $P_{0}=\left\{p_{u}(0) \mid x \in K\right.$ and $\left.S(x)=C(u)\right\}$ is compact in $\mathbb{R}^{n}$.

The proof of this fact is a consequence of the continuity of the extremal flow (for more details see [33, Lemma 4.9]).

Let us end the proof of Lemma 3.7. Let $A$ be a subanalytic compact subset of $\mathbb{R}^{n}$ containing the set $P_{0}$. Using the mapping $\Phi$ defined as (40), for every $x \in K$ we can express the final time $t(x, u)=\inf \left\{t \mid E_{x, t}(u) \in \Sigma\right\}$ restricted to minimizing controls as a function of $p_{0}$

$$
\tau\left(x, p_{0}\right)=t\left(x, \Phi\left(p_{0}\right)\right)=\inf \left\{t \mid\left(E_{x, t} \circ \Phi\right)\left(p_{0}\right) \in \Sigma\right\},
$$

where $p_{0} \in A$. From Proposition 1.2, this mapping is subanalytic on $K \times A$. Let us further express in this way the value function

$$
S(x)=\inf \left\{C(u) \mid E_{x, t(x, u)}(u) \in \Sigma\right\} .
$$

To this aim, set

$$
\mathcal{C}\left(x, p_{0}\right)=C\left(\Phi\left(p_{0}\right)\right) .
$$

The function $\mathcal{C}$ is clearly subanalytic on $K \times A$. We have

$$
S(x)=\inf \left\{\mathcal{C}\left(x, p_{0}\right) \mid p_{0} \in A \cap\left(E_{x, \tau\left(x, p_{0}\right)} \circ \Phi\right)^{-1}(\Sigma)\right\},
$$

and Proposition 1.2 implies that $S$ is subanalytic on $K$.

To end the proof of Theorem 3.1, it remains to prove that $S$ is a viscosity solution of the Dirichlet problem (31). Since $S$ is continuous from Lemma 3.6, this is a consequence of [9, Proposition 3.12, p. 255].

\subsubsection{Proof of Proposition 3.2}

Let us prove that $S$ is upper and lower semi-continuous on $\bar{\Omega}$.

Lemma 3.8. The function $S$ is upper semi-continuous on $\bar{\Omega}$.

Proof. Let $z \in \Sigma$ be fixed. We have to prove that

$$
\limsup _{x \in \Omega, x \rightarrow z} S(x) \leqslant S(z)=g(z) .
$$

For every $x \in \Omega$, by definition,

$$
S(x)=\inf \left\{\int_{0}^{t(x, u)}\left(\sum_{i=1}^{m} u_{i}(s)^{2}+c\right) \mathrm{d} s+g\left(x_{u}(t(x, u))\right) \mid x_{u}(0)=x\right\} .
$$

On the other part, the compatibility condition (30) implies that, for every control $u$, and every $t>t(x, u)$ such that $x_{u}(t) \in \Sigma$ and $x_{u}(s) \in \bar{\Omega}, s \in[0, t]$, there holds

$$
\int_{0}^{t(x, u)}\left(\sum_{i=1}^{m} u_{i}(s)^{2}+c\right) \mathrm{d} s+g\left(x_{u}(t(x, u))\right) \leqslant \int_{0}^{t}\left(\sum_{i=1}^{m} u_{i}(s)^{2}+c\right) \mathrm{d} s+g\left(x_{u}(t)\right) .
$$


Hence,

$$
S(x)=\inf \left\{\int_{0}^{t}\left(\sum_{i=1}^{m} u_{i}(s)^{2}+c\right) \mathrm{d} s+g\left(x_{u}(t)\right) \mid t>0, x_{u}(0)=x, x_{u}(t) \in \Sigma, \text { and } x_{u}(s) \in \bar{\Omega}, \forall s \in[0, t]\right\} .
$$

For all $x \in \bar{\Omega}, y \in \Sigma$, and $t>0$, define

$$
S(t, x, y)=\inf \left\{\int_{0}^{t} \sum_{i=1}^{m} u_{i}(s)^{2} \mathrm{~d} s \mid x_{u}(0)=x, x_{u}(t)=y\right\}
$$

(with the agreement that $S(t, x, y)=+\infty$ if there does not exist any trajectory $x_{u}(\cdot)$ joining $x$ to $y$ ), so that

$$
S(x)=\inf \{S(t, x, y)+c t+g(y) \mid t>0, y \in \Sigma\} .
$$

In particular we have for all $t>0$

$$
S(x) \leqslant S(t, x, z)+c t+g(z) .
$$

On the other part, we claim that there exist a neighborhood of $z$ in $\bar{\Omega}$ and positive real numbers $\alpha_{1}, \alpha_{2}$, such that if $t>0$ is small enough then

$$
S(t, x, z) \leqslant \frac{d_{S R}(x, z)^{2}}{t}+\alpha_{1} d_{S R}(x, z)+\alpha_{2} t,
$$

where $d_{S R}(\cdot, \cdot)$ denotes the sub-Riemannian distance associated to the $m$-uple of vector fields $\left(f_{1}, \ldots, f_{m}\right)$. This inequality is proved apart in the next lemma. From (46) and (47), we infer easily

$$
\limsup _{x \in \Omega, x \rightarrow z} S(x) \leqslant g(z) \text {. }
$$

Lemma 3.9. For every $z \in \Sigma$, there exist a neighborhood $V$ of $z$ and positive real numbers $\varepsilon, \alpha_{1}, \alpha_{2}$, such that, for every $x \in V \cap \bar{\Omega}$, and every $t \in] 0, \varepsilon[$, there holds

$$
S(t, x, z)=\frac{d_{S R}(x, z)^{2}}{t}+R(t, x)
$$

with

$$
|R(t, x)| \leqslant \alpha_{1} d_{S R}(x, z)+\alpha_{2} t .
$$

Proof. Let $z \in \Sigma$ be fixed. First of all, it was already noticed in Remark 3.6 that assumption (32) implies that the control affine system (24) is small-time locally controllable near $\Sigma$, and thus, if $x$ is close enough to $z$ and $t>0$ is small enough, then $S(t, x, z)<+\infty$. On the other part, under assumption (32) the sub-Riemannian distance $x \mapsto$ $d_{S R}(x, z)$ associated to the $m$-uple of vector fields $\left(f_{1}, \ldots, f_{m}\right)$ is well defined in a neighborhood of $z$.

For every solution $x_{u}(\cdot)$ of the affine system (24) defined on $[0, t]$, steering a point $x \in V$ to $z \in \Sigma$, set

$$
\forall s \in[0,1] \quad \tilde{x}(s)=x_{u}(s t) \quad \text { and } \quad \tilde{u}_{i}(s)=t u_{i}(t s), \quad i=1, \ldots, m .
$$

Then,

$$
\tilde{x}^{\prime}(s)=t f_{0}(\tilde{x}(s))+\sum_{i=1}^{m} \tilde{u}_{i}(s) f_{i}(\tilde{x}(s)) \quad \text { a.e. on }[0,1],
$$

and $\tilde{x}(0)=x, \tilde{x}(1)=z$. If the parameter $t>0$ is small, the control system (48) can be considered as a perturbation of the sub-Riemannian system associated to $\left(f_{1}, \ldots, f_{m}\right)$.

By hypothesis, minimizing trajectories solutions of (48), steering a point $x \in V \cap \Omega$ to $z \in \Sigma$, cannot be singular, and thus are associated to normal controls. Hence, from the Pontryagin Maximum Principle, we have, for all $i \in$ $\{1, \ldots, m\}$ and $s \in[0,1]$,

$$
\tilde{u}_{i}(s)=\left\langle\tilde{p}(s), f_{i}(\tilde{x}(s))\right\rangle \text {, }
$$


where $\tilde{p}(\cdot):[0,1] \rightarrow \mathbb{R}^{n}$ is an absolutely continuous function satisfying

$$
\tilde{p}^{\prime}(s)=-\tilde{p}(s)\left(t \frac{\partial f_{0}}{\partial x}(\tilde{x}(s))+\sum_{i=1}^{m} \tilde{u}_{i}(s) \frac{\partial f_{i}}{\partial x}(\tilde{x}(s))\right) \quad \text { a.e. on }[0,1] .
$$

When $t=0$, the system (48), (49) still makes sense, and parametrizes normal extremals steering $x$ to $z$ for the subRiemannian structure associated to the $m$-uple $\left(f_{1}, \ldots, f_{m}\right)$. As this system depends analytically on the parameter $t$ and on the initial condition $x$, up to reducing $V$ we can write, for every $i \in\{1, \ldots, m\}$,

$$
\tilde{u}_{i}(s)=\tilde{u}_{i}^{0}(s)+t r_{i}(s),
$$

where $r_{i}(\cdot)$ is uniformly bounded as $s \in[0,1], t \in[0, \varepsilon]$ and $x \in V$. Hence,

$$
t \int_{0}^{t} \sum_{i=1}^{m} u_{i}(\tau)^{2} \mathrm{~d} \tau=\int_{0}^{1} \sum_{i=1}^{m} \tilde{u}_{i}(s)^{2} \mathrm{~d} s=\int_{0}^{1} \sum_{i=1}^{m} \tilde{u}_{i}^{0}(s)^{2} \mathrm{~d} s+2 t \int_{0}^{1} \sum_{i=1}^{m} \tilde{u}_{i}^{0}(s) r_{i}(s) \mathrm{d} s+t^{2} \int_{0}^{1} \sum_{i=1}^{m} r_{i}(s)^{2} \mathrm{~d} s,
$$

from which it is not difficult to infer that

$$
t S(t, x, z)=d_{S R}(x, z)^{2}+t R(t, x),
$$

where $R(t, x)$ satisfies the inequality announced in the statement of the lemma.

Lemma 3.10. The function $S$ is lower semi-continuous on $\bar{\Omega}$.

Proof. We have to prove that, for every $x \in \Sigma$, and every sequence $\left(x_{n}\right)_{n \in \mathbb{N}}$ of points of $\Omega$ converging towards $x$, there holds

$$
S(x) \leqslant \liminf S\left(x_{n}\right) .
$$

Let us notice again that assumption (32) implies that the affine control system (24) is locally controllable near $\Sigma$, hence clearly the sequence $\left(S\left(x_{n}\right)\right)_{n \in \mathbb{N}}$ is bounded. A reasoning similar to proof of Lemma 3.6 permits to conclude.

Lemmas 3.8 and 3.10 prove the continuity of $S$ on the whole $\bar{\Omega}$. Uniqueness is then a consequence of [10, Theorem 2.7, p. 37] or [9, Proposition 3.13, p. 256].

\subsubsection{Proof of Proposition 3.3}

For every $x \in \Omega$, there exists a minimizing control $u$ (not necessarily unique) steering $x$ to $\Sigma$ in time $t(x, u)$. By the choice axiom, we construct a function $\tau(\cdot)$ on $\Omega$ which to $x \in \Omega$ associates $\tau(x)=t(x, u)$ for a choice of such a control $u$. The following lemma is a consequence of the strict compatibility inequality which is now assumed.

Lemma 3.11. Under the strict compatibility assumption, the time $\tau(x)$ tends to 0 as $x \in \Omega$ tends to $\Sigma$.

Proof. If not, there exist $x \in \Sigma$ and a sequence $\left(x_{n}\right)_{n \in \mathbb{N}}$ of points of $\Omega$ converging to $x$ such that $\tau\left(x_{n}\right)$ does not tend to 0 . Since $S$ is continuous on $\bar{\Omega}$ from Proposition 3.2, the sequence $\left(\tau\left(x_{n}\right)\right)_{n \in \mathbb{N}}$ is clearly bounded, and hence, up to a subsequence, it converges to a real $t>0$. For every integer $n$, let $u_{n}$ be a minimizing control steering $x_{n}$ to $\Sigma$ in time $t\left(x_{n}, u_{n}\right)=\tau\left(x_{n}\right)$, and let $x_{u_{n}}(\cdot)$ denote the associated trajectory. As in the proof of Lemma 3.7, we can assume that $u_{n}$ tends to a control $u$ in weak $L^{2}$-topology, and this yields the inequality

$$
\int_{0}^{t}\left(\sum_{i=1}^{m} u_{i}(s)^{2}+c\right) \mathrm{d} s+g\left(x_{u}(t)\right) \leqslant \liminf S\left(x_{n}\right)=S(x)=g(x) .
$$

Moreover, $x_{u_{n}}(\cdot)$ tends uniformly to $x_{u}(\cdot)$, and hence $x_{u}(\cdot)$ is a trajectory contained in $\bar{\Omega}$ steering $x \in \Sigma$ to $x_{u}(t) \in \Sigma$. According to the strict compatibility assumption, there must hold

$$
g(x)-g\left(x_{u}(t)\right)<\int_{0}^{t}\left(\sum_{i=1}^{m} u_{i}(s)^{2}+c\right) \mathrm{d} s,
$$

and this contradicts inequality (50). 
For every $x \in \Omega$, there exists a minimizing control $u$ steering $x$ to $\Sigma$ in time $\tau(x)$, and the associated trajectory $x_{u}(\cdot)$ satisfies

$$
\dot{x}_{u}(t)=f_{0}\left(x_{u}(t)\right)+\sum_{i=1}^{m} u_{i}(t) f_{i}\left(x_{u}(t)\right) \quad \text { a.e. on }[0, \tau(x)] .
$$

As in the proof of Lemma 3.9, let us reparametrize this control system on $[0,1]$, by setting, for every $s \in[0,1]$,

$$
\tilde{x}(s)=x_{u}(s \tau(x)) \quad \text { and } \quad \tilde{u}_{i}(s)=\tau(x) u_{i}(s \tau(x)), \quad i=1, \ldots, m,
$$

so that every extremal is solution of the system

$$
\begin{aligned}
& \tilde{x}^{\prime}(s)=\tau(x) f_{0}(\tilde{x}(s))+\sum_{i=1}^{m} \tilde{u}_{i}(s) f_{i}(\tilde{x}(s)), \\
& \tilde{p}^{\prime}(s)=-\tilde{p}(s)\left(\tau(x) \frac{\partial f_{0}}{\partial x}(\tilde{x}(s))+\sum_{i=1}^{m} \tilde{u}_{i}(s) \frac{\partial f_{i}}{\partial x}(\tilde{x}(s))\right),
\end{aligned}
$$

with

$$
\tilde{u}_{i}(s)=\tilde{p}(s) f_{i}(\tilde{x}(s)), \quad i=1, \ldots, m .
$$

One has to prove that the set of vectors $\tilde{p}(0)$ such that the associated trajectory steers $x$ to $\Sigma$, is compact as $x$ varies in $\bar{\Omega}$. Of course a problem arises when the point $x \in \Omega$ tends to $\Sigma$, and in this case $\tau(x) \rightarrow 0$. At the limit one recovers a sub-Riemannian structure associated to the $m$-uple $\left(f_{1}, \ldots, f_{m}\right)$, which is medium-fat by assumption. In this case, we know from [3, Proof of Theorem 6] (see also [1, Theorem 5]) that the set of initial adjoint vectors is compact. Since our system is a perturbation of this sub-Riemannian structure as $\tau(x)$ tends to 0 , we infer the desired compactness property.

The end of the proof is then strictly similar to the proof of Lemma 3.7.

\subsection{The Cauchy problem}

\subsubsection{Statement of the results}

We first investigate Cauchy problems in the whole $\mathbb{R}^{n}$.

Theorem 3.12. Let $f_{0}, \ldots, f_{m}$ be analytic vector fields on $\mathbb{R}^{n}$, and let $H(x, p)$ be the Hamiltonian function defined on $\mathbb{R}^{n} \times \mathbb{R}^{n}$ by

$$
H(x, p)=\left\langle p, f_{0}(x)\right\rangle+\sum_{i=1}^{m}\left\langle p, f_{i}(x)\right\rangle^{2} .
$$

Let $T>0$ be fixed, and let $g$ be a subanalytic function on $\mathbb{R}^{n}$. For all $x \in \mathbb{R}^{n}$ and $t \in[0, T]$, consider the problem of determining a solution of the affine control system

$$
x_{u}^{\prime}(s)=f_{0}\left(x_{u}(s)\right)+\sum_{i=1}^{m} u_{i}(s) f_{i}\left(x_{u}(s)\right) \text { a.e. on }[0, t],
$$

such that

$$
x_{u}(t)=x,
$$

minimizing the cost

$$
C(t, u)=\int_{0}^{t} \sum_{i=1}^{m} u_{i}(s)^{2} \mathrm{~d} s+g\left(x_{u}(0)\right) .
$$

We assume that, for every $x \in \mathbb{R}^{n}$, there exists a control $u=\left(u_{1}, \ldots, u_{m}\right) \in L^{2}\left([0, T], \mathbb{R}^{m}\right)$ such that the associated trajectory $x_{u}(\cdot)$ is well defined on $[0, T]$, and satisfies $x_{u}(T)=x$. 
Let $S(t, x)$ denote the value function associated to the optimal control problem (51)-(53). Namely, if $\mathcal{S}_{t}$ denotes the set of couples $\left(x_{u}(\cdot), u(\cdot)\right)$ solutions of $(51)$, one has, for all $t \in[0, T]$ and $x \in \mathbb{R}^{n}$,

$$
S(t, x)=\inf \left\{C(t, u) \mid\left(x_{u}(\cdot), u(\cdot)\right) \in \mathcal{S}_{t}, x_{u}(t)=x\right\} .
$$

If $g$ is proper, and if, for all $x \in \mathbb{R}^{n}$ and $\left.\left.t \in\right] 0, T\right]$, there exists no singular minimizing trajectory of the optimal control problem (51)-(53), then $S$ is continuous and subanalytic on $] 0, T] \times \mathbb{R}^{n}$, and is solution of the Cauchy problem

$$
\begin{aligned}
& \left.\left.\frac{\partial S}{\partial t}+H\left(x, \frac{\partial S}{\partial x}\right)=0 \quad \text { a.e. in }\right] 0, T\right] \times \mathbb{R}^{n}, \\
& S(0, \cdot)=g(\cdot) .
\end{aligned}
$$

Remark 3.14. Since $S$ is subanalytic on $] 0, T] \times \mathbb{R}^{n}$, it is almost everywhere differentiable, and thus (55) makes sense.

Remark 3.15. There is a priori no guarantee that the function $S$ be a viscosity solution on the one part, and that $S$ be the unique solution on the other part.

Remark 3.16. The assumption of well defined trajectories is satisfied if for instance the vector field $f_{0}$ is complete.

Remark 3.17. Remark 3.3 on genericity holds again here.

Proposition 3.13. Under the assumptions of Theorem 3.12, if there holds moreover

$$
\operatorname{Lie}\left(f_{1}(x), \ldots, f_{m}(x)\right)=\mathbb{R}^{n}
$$

for every $x \in \mathbb{R}^{n}$, then $S$ is continuous and subanalytic on $[0, T] \times \mathbb{R}^{n}$.

We next investigate Cauchy-Dirichlet problems on a subset $\Omega$ of $\mathbb{R}^{n}$.

Theorem 3.14. Let $\Omega$ be a bounded analytic open subset of $\mathbb{R}^{n}$. Consider the Hamiltonian function on $\Omega \times \mathbb{R}^{n}$ defined by

$$
H(x, p)=\left\langle p, f_{0}(x)\right\rangle+\sum_{i=1}^{m}\left\langle p, f_{i}(x)\right\rangle^{2},
$$

where $f_{0}, \ldots, f_{m}$ are analytic vector fields on $\mathbb{R}^{n}$. Let $\Sigma=\partial \Omega, T>0$ be fixed and $g$ be a subanalytic function on $[0, T] \times \Sigma$. Consider the affine control system

$$
x_{u}^{\prime}(s)=f_{0}\left(x_{u}(s)\right)+\sum_{i=1}^{m} u_{i}(s) f_{i}\left(x_{u}(s)\right)
$$

and the cost

$$
C(t, u)=\int_{0}^{t} \sum_{i=1}^{m} u_{i}(s)^{2} \mathrm{~d} s+g\left(t, x_{u}(t)\right) .
$$

Assume that, for every $t \in] 0, T]$,

(1) The boundary $\Sigma$ is accessible from $\Omega$ in time $t$, i.e., for every time $t \in] 0, T]$, and every $x \in \Omega$, there exists a control $u$ on $[0, t]$ such that the associated solution $x_{u}(\cdot)$ of $(56)$ satisfies $x_{u}(0)=x$ and $x_{u}(t) \in \Sigma$.

(2) There exists no singular minimizing trajectory for the optimal control problem (56), (57), steering $\Omega$ to $\Sigma$ in time $t$.

For every $t \in] 0, T]$ and every $x \in \bar{\Omega}$, let $S(t, x)$ be the value function associated to the optimal control problem of determining a trajectory solution of the control system (56) on $[0, t]$, minimizing the cost (57), and such that $x_{u}(0)=x$ and $x_{u}(t) \in \Sigma$. Namely, if $\mathcal{S}$ denotes the set of couples $\left(u(\cdot), x_{u}(\cdot)\right)$ solutions of the control system (56), one has

$$
S(t, x)=\inf \left\{C(t, u) \mid\left(x_{u}(\cdot), u(\cdot)\right) \in \mathcal{S}, x_{u}(0)=x, x_{u}(t) \in \Sigma\right\} .
$$


For all $s, t \in[0, T]$ such that $s<t$, and all $x \in \Sigma, y \in \bar{\Omega}$, set

$$
S(t, x, s, y)=\inf \left\{\int_{s}^{t} \sum_{i=1}^{m} u_{i}(\tau)^{2} \mathrm{~d} \tau \mid\left(x_{u}(\cdot), u(\cdot)\right) \in \mathcal{S}, x_{u}(s)=y, x_{u}(t)=x\right\} .
$$

Assume that $g$ satisfies the compatibility condition

$$
\forall(s, y) \in(\{0\} \times \bar{\Omega}) \cup([0, T[\times \partial \Omega) \forall(t, x) \in] s, T] \times \partial \Omega \quad g(t, x)-g(s, y) \leqslant S(t, x, s, y) .
$$

Then, $S$ is continuous and subanalytic on $] 0, T] \times \Omega$, and is a viscosity solution of the Cauchy-Dirichlet problem

$$
\begin{aligned}
& \left.\left.\frac{\partial S}{\partial t}+H\left(x, \frac{\partial S}{\partial x}\right)=0 \quad \text { in }\right] 0, T\right] \times \Omega, \\
& S=g \quad \text { on }] 0, T] \times \partial \Omega, \\
& \lim _{t \rightarrow 0} S(t, x)=g(0, x) \quad \text { in } \Omega .
\end{aligned}
$$

Remark 3.18. Remark 3.3 on genericity holds again here.

Proposition 3.15. Under the assumptions of Theorem 3.14, if there holds moreover

$$
\operatorname{Lie}\left(f_{1}(x), \ldots, f_{m}(x)\right)=\mathbb{R}^{n}
$$

for every $x \in \bar{\Omega}$, then $S$ is continuous on $[0, T] \times \bar{\Omega}$. As a consequence, $S$ is the unique viscosity solution of the Cauchy-Dirichlet problem (59).

Proposition 3.16. Under the assumptions of Theorem 3.14, if the m-uple of vector fields $\left(f_{1}, \ldots, f_{m}\right)$ is moreover medium-fat on $\bar{\Omega}$, then $S$ is subanalytic on $[0, T] \times \bar{\Omega}$.

\subsubsection{Proof of Theorem 3.12}

The proof is similar to the proof of Theorem 3.1 and is only sketched. We proceed in four steps.

Step 1. The infimum of formula (54) is a minimum, i.e. there exist minimizing trajectories for the optimal control problem (51)-(53).

Indeed, let $\left(\left(u_{i}^{n}\right)_{1 \leqslant i \leqslant n}, x_{0}^{n}\right)_{n \in \mathbb{N}}$ be a sequence converging to the infimum, and, for every $n$, let $x_{n}(\cdot)$ denote the corresponding trajectory, with $x_{n}(0)=x_{0}^{n}$. Since $g$ is proper, we infer that the sequence $\left(x_{0}^{n}\right)_{n \in \mathbb{N}}$ is bounded in $\mathbb{R}^{n}$ (and thus, resp., $\left(u_{i}^{n}\right)_{n \in \mathbb{N}}$ is bounded in $L^{2}([0, t], \mathbb{R})$, for every $\left.i \in\{1, \ldots, m\}\right)$, and hence up to a subsequence it converges to a point $x_{0} \in \mathbb{R}^{n}$ (resp., converges weakly to a control $u_{i} \in L^{2}([0, t], \mathbb{R}$ ), for every $i \in\{1, \ldots, m\}$ ). Therefore, the sequence of curves $x_{n}(\cdot)$ converges uniformly to $x_{u}(\cdot)$ on $[0, t]$, and in particular $x_{u}(0)=x_{0}$ and $x_{u}(t)=x$. Moreover, one has

$$
\int_{0}^{t} \sum_{i=1}^{m} u_{i}(s)^{2} \mathrm{~d} s+g\left(x_{0}\right) \leqslant \liminf _{n \rightarrow+\infty} \int_{0}^{t} \sum_{i=1}^{m} u_{i}^{n}(s)^{2} \mathrm{~d} s+g\left(x_{0}^{n}\right)=S(t, x),
$$

and this inequality is necessarily an equality. Hence, $x_{u}(\cdot)$ is minimizing.

Step 2. We write $S(t, x)$ so that Proposition 1.2 shall be applied. In the same way as in the proof of Theorem 3.1, an application of the Pontryagin Maximum Principle leads to

$$
S(t, x)=\inf \left\{\psi\left(t, x_{0}, p_{0}\right) \mid \varphi\left(t, x_{0}, p_{0}\right)=x\right\},
$$

where $\varphi$ and $\psi$ are analytic mappings on $] 0, T] \times \mathbb{R}^{n} \times \mathbb{R}^{n}$.

Step 3. The value function $S$ is continuous on $] 0, T] \times \mathbb{R}^{n}$.

The proof is similar to Lemma 3.6 and is skipped.

Step 4. $S$ is subanalytic on $] 0, T] \times \mathbb{R}^{n}$.

Indeed, let $K$ be a compact subanalytic subset of $] 0, T] \times \mathbb{R}^{n}$. To prove that $S$ is subanalytic on $K$, similarly to Lemma 3.7, it suffices to show that the set

$$
P_{0}=\left\{\left(x_{0}, p_{0}\right) \in \mathbb{R}^{n} \times \mathbb{R}^{n} \mid \exists(t, x) \in K \varphi\left(t, x_{0}, p_{0}\right)=x\right\}
$$


is compact.

On the one part, since the initial point is not fixed, all extremals of the problem can be chosen so as to be normal, and moreover

$$
p(0)=-\frac{1}{2} \nabla g\left(x_{0}\right) .
$$

On the other part, $S$ is continuous and thus bounded on $K$. Since $g$ is proper, the compactness of $P_{0}$ follows easily. An application of Proposition 1.2 ends the proof.

Remark 3.19. The assumption of nonexistence of singular minimizing trajectories cannot be skipped in Theorem 3.12. Indeed it permits to prove that the value function $S$ is continuous (actually that $S$ is upper semi-continuous; it is indeed always lower semi-continuous), and this fact is essential in Step 4 to assert that $S$ has an upper bound on the compact $K$.

\subsubsection{Proof of Proposition 3.13}

Regarding Remark 3.19, it suffices to show that $S$ is continuous as $t$ tends to 0 . Similarly to Lemma 3.9, one can prove, for $t>0$ small enough, that

$$
S(t, x)=\inf _{y \in \mathbb{R}^{n}}\left(\frac{d_{S R}(x, y)^{2}}{t}+R(t, x)+g(y)\right),
$$

where $R$ is uniformly bounded, and $d_{S R}(\cdot, \cdot)$ is the sub-Riemannian distance associated to the $m$-uple of vector fields $\left(f_{1}, \ldots, f_{m}\right)$. It follows easily from $(62)$ that

$$
\lim _{t \rightarrow 0} S(t, x)=g(x)
$$

\subsubsection{Proof of Theorem 3.14}

The proof is similar (and simpler, for the final time is fixed) to the proof of Theorem 3.1, and is skipped.

\subsubsection{Proof of Proposition 3.15}

The proof of the continuity of $S$ as $t$ tends to 0 is the same as in Proposition 3.13. Outside $t=0$ the proof is a little more intricate. What follows is quite analogous to [27, pp. 219-220]. Let $\left.\left.\left(t_{0}, x_{0}\right) \in\right] 0, T\right] \times \Sigma$. We next prove that $S(t, x) \rightarrow S\left(t_{0}, x_{0}\right)=g\left(t_{0}, x_{0}\right)$ as $(t, x) \rightarrow\left(t_{0}, x_{0}\right)$. First of all, notice that

$$
S(t, x)=\inf \{S(t, x, s, y)+g(s, y) \mid(s, y) \in(\{0\} \times \bar{\Omega}) \cup([0, t[\times \Sigma)\} .
$$

We proceed in two steps.

First step: $S$ is upper semi-continuous at $\left(t_{0}, x_{0}\right)$.

Indeed, since $x_{0} \in \Sigma$, one has $S(t, x) \leqslant g\left(s, x_{0}\right)+S\left(t, x, s, x_{0}\right)$, for every $s \in\left[0, t_{0}\right.$ [. Moreover, similarly to Lemma 3.9,

$$
S\left(t, x, s, x_{0}\right) \leqslant \frac{d_{S R}\left(x_{0}, x\right)^{2}}{t-s}+\alpha_{1} d_{S R}\left(x_{0}, x\right)+\alpha_{2}(t-s),
$$

for every $x \in \bar{\Omega}$, if $t-s>0$ is small enough, where $\alpha_{1}, \alpha_{2} \geqslant 0$. We then infer easily that

$$
\limsup _{(t, x) \rightarrow\left(t_{0}, x_{0}\right)} S(t, x) \leqslant g\left(t_{0}, x_{0}\right) .
$$

Second step: $S$ is lower semi-continuous at $\left(t_{0}, x_{0}\right)$.

Indeed, let $\left(t^{n}, x^{n}\right)_{n \in \mathbb{N}}$ be a sequence of $\left.] 0, T\right] \times \Omega$ converging to $\left(t_{0}, x_{0}\right)$. From (63), there exists a sequence $\left(s^{n}, y^{n}\right)_{n \in \mathbb{N}}$ of $(\{0\} \times \bar{\Omega}) \cup([0, T] \times \Sigma)$ such that $s^{n}<t^{n}$ for every integer $n$, and

$$
S\left(t^{n}, x^{n}\right)-g\left(s^{n}, y^{n}\right)-S\left(t^{n}, x^{n}, s^{n}, y^{n}\right) \underset{n \rightarrow+\infty}{\longrightarrow} 0 .
$$

Up to a subsequence, we can suppose that $\left(s^{n}, y^{n}\right)$ tends to $\left(s_{0}, y_{0}\right) \in(\{0\} \times \bar{\Omega}) \cup\left(\left[0, t_{0}\right] \times \Sigma\right)$. Three cases occur: 
1. If $s_{0}<t_{0}$, using the assumption of the absence of singular minimizers, it is easy to prove that

$$
S\left(t^{n}, x^{n}, s^{n}, y^{n}\right) \underset{n \rightarrow+\infty}{\longrightarrow} S\left(t_{0}, x_{0}, s_{0}, y_{0}\right) .
$$

Hence

$$
\liminf _{n \rightarrow+\infty} S\left(t^{n}, x^{n}\right) \geqslant g\left(s_{0}, y_{0}\right)+S\left(t_{0}, x_{0}, s_{0}, y_{0}\right),
$$

and applying the compatibility condition (58), we infer

$$
\liminf _{n \rightarrow+\infty} S\left(t^{n}, x^{n}\right) \geqslant g\left(t_{0}, x_{0}\right) .
$$

2. If $s_{0}=t_{0}$ and $x_{0}=y_{0}$, we have obviously

$$
\liminf _{n \rightarrow+\infty} S\left(t^{n}, x^{n}\right) \geqslant g\left(t_{0}, x_{0}\right)
$$

since $S\left(t^{n}, x^{n}, s^{n}, y^{n}\right) \geqslant 0$ for every integer $n$.

3. If $s_{0}=t_{0}$ and $x_{0} \neq y_{0}$, then $t^{n}-s^{n}$ tends to 0 , and, similarly to Lemma 3.9, one can prove the existence of nonnegative real numbers $\alpha_{1}, \alpha_{2}$, such that

$$
S\left(t^{n}, x^{n}, s^{n}, y^{n}\right) \geqslant \frac{d_{S R}\left(x^{n}, y^{n}\right)^{2}}{t^{n}-s^{n}}-\alpha_{1} d_{S R}\left(x^{n}, y^{n}\right)-\alpha_{2}\left(t^{n}-s^{n}\right),
$$

for every integer $n$. Hence, $S\left(t^{n}, x^{n}, s^{n}, y^{n}\right) \underset{n \rightarrow+\infty}{\longrightarrow}+\infty$ for $d_{S R}\left(x_{0}, y_{0}\right) \neq 0$. In particular, $S\left(t^{n}, x^{n}\right) \underset{n \rightarrow+\infty}{\longrightarrow}+\infty$. On the other part, notice that, due to the assumption (60), one can easily prove that the value function $S$ is bounded on each $[\varepsilon, T] \times \bar{\Omega}$, where $\varepsilon>0$. This is a contradiction.

\subsection{Further comments}

It is rather clear how to extend the previous results to more general situations. Indeed, notice the three following facts.

1. First of all, using notations of Section 3.1, the author provides in [34] some general conditions on the control system (22) and on the cost (23) ensuring that the associated value function is subanalytic. The main assumption is the absence of singular minimizing trajectories.

2. On the other part, it is well known that, under some general assumptions, the previous value function is a viscosity solution of the Hamilton-Jacobi equation

$$
\frac{\partial v}{\partial t}+H_{1}\left(x, \frac{\partial v}{\partial x}\right)=0,
$$

where $H_{1}(x, p)=\max _{u} H(x, p, u)$. Notice that all comments here also hold in the Dirichlet case where the value function does not depend on $t$.

3. Finally, in [25,29], the authors prove that, under general assumptions on the Hamiltonian $H_{1}$, there exists an optimal control problem such that the associated value function is exactly the viscosity solution of (64) (inverse optimal control problem). Their proof can be quite readily extended to the subanalytic case.

Gathering these facts leads to a general statement ensuring that the unique viscosity solution of an HamiltonJacobi equation is subanalytic, provided that the associated optimal control problem would not admit any singular minimizing trajectory.

However, the proof of the third fact, mainly based on Kakutani Fixed Point Theorem, is not constructive. Hence, in general, it may be difficult to check whether or not an underlying optimal control problem admits some singular minimizing trajectories.

We conclude with the following question: given an analytic function $H_{1}(x, p)$, convex in $p$ (but not strictly convex), is it possible to set some conditions ensuring that an associated subanalytic optimal control problem does not admit any singular minimizing trajectory? 


\section{Acknowledgement}

I wish to thank P. Gérard for his reading and advice, and also A. Fathi for his interest to this work.

\section{References}

[1] A. Agrachev, Compactness for sub-Riemannian length minimizers and subanalyticity, Rend. Sem. Mat. Torino 56 (1999).

[2] A. Agrachev, B. Bonnard, M. Chyba, I. Kupka, Sub-Riemannian sphere in the Martinet flat case, ESAIM Control Optim. Calc. Var. 2 (1997) $377-448$.

[3] A. Agrachev, J.P. Gauthier, On subanalyticity of Carnot-Carathéodory distances, Ann. Inst. H. Poincaré Anal. Non Linéaire 18 (2001) 3.

[4] A. Agrachev, A. Sarychev, Sub-Riemannian metrics: minimality of singular geodesics versus subanalyticity, ESAIM Control Optim. Calc. Var. 4 (1999) 377-403.

[5] P. Albano, P. Cannarsa, Structural properties of singularities of semiconcave functions, Ann. Scuola Norm. Sup. Pisa Cl. Sci. (4) 28 (4) (1999) 719-740.

[6] G. Alberti, L. Ambrosio, P. Cannarsa, On the singularities of convex functions, Manuscripta Math. 76 (3-4) (1992) $421-435$.

[7] F. Alouges, S. Labbé. Z-invariant micromagnetic configurations, Preprint Univ. Paris Sud, Orsay, 2005.

[8] L. Ambrosio, P. Cannarsa, H.M. Soner, On the propagation of singularities of semi-convex functions, Ann. Scuola Norm. Sup. Pisa Cl. Sci. (4) 20 (4) (1993) 597-616.

[9] M. Bardi, I. Capuzzo-Dolcetta, Optimal Control and Viscosity Solutions of Hamilton-Jacobi-Bellman Equations, Birkhäuser, Boston, 1997.

[10] G. Barles, Solutions de viscosité des équations de Hamilton-Jacobi, Math. Appl., vol. 17, Springer-Verlag, 1994.

[11] A. Bellaïche, Tangent space in sub-Riemannian geometry, in: Sub-Riemannian Geometry, Birkhäuser, 1996.

[12] B. Bonnard, M. Chyba, Singular trajectories and their role in control theory, Math. Appl. 40 (2003).

[13] B. Bonnard, E. Trélat, On the role of singular minimizers in sub-Riemannian geometry, Ann. Fac. Sci. Toulouse (6) X (3) (2001) 405-491.

[14] P. Cannarsa, A. Mennucci, C. Sinestrari, Regularity results for solutions of a class of Hamilton-Jacobi equations, Arch. Rational Mech. Anal. 40 (1997) 197-223.

[15] P. Cannarsa, C. Sinestrari, Convexity properties of the minimum time function, Calc. Var. Partial Differential Equations 3 (1995) $273-298$.

[16] P. Cannarsa, C. Sinestrari, Semiconcave Functions, Hamilton-Jacobi Equations, and Optimal Control, Progr. Nonlinear Differential Equations Appl., vol. 58, Birkhäuser, Boston, 2004.

[17] P. Cannarsa, H.M. Soner, On the singularities of the viscosity solutions to Hamilton-Jacobi-Bellman equations, Indiana Univ. Math. J. 36 (3) (1987) 501-524.

[18] Y. Chitour, F. Jean, E. Trélat, Propriétés génériques des trajectoires singulières, C. R. Acad. Sci. Paris, Ser. I 337 (1) (2003) $49-52$.

[19] Y. Chitour, F. Jean, E. Trélat. Genericity properties for singular trajectories, J. Differential Geom. (2005), in press.

[20] M.G. Crandall, P.-L. Lions, Viscosity solutions of Hamilton-Jacobi equations, Trans. Amer. Math. Soc. 277 (1983) 1-42.

[21] L.C. Evans, Partial Differential Equations, Amer. Math. Soc., 1998.

[22] A. Fathi, Weak KAM Theorem and Lagrangian Dynamics, Cambridge University Press, 2003.

[23] R.M. Hardt, Stratification of real analytic mappings and images, Invent. Math. 28 (1975).

[24] H. Hironaka, Subanalytic sets, in: Number Theory, Algebraic Geometry and Commutative Algebra. In honor of Y. Akizuki, Tokyo, 1973.

[25] H. Ishii, On representation of solutions of Hamilton-Jacobi equations with convex Hamiltonians, in: Lecture Notes Numer. Appl. Anal., vol. 8, 1985, pp. 15-22.

[26] V. Jurdjevic, Geometric Control Theory, Cambridge University Press, 1997.

[27] P.-L. Lions, Generalized Solutions of Hamilton-Jacobi Equations, Pitman, 1982.

[28] L.S. Pontryagin, V.G. Boltyanskij, R.V. Gamkrelidze, E.F. Mishchenko, The Mathematical Theory of Optimal Processes, Interscience Publishers, John Wiley \& Sons, New York, 1962.

[29] F. Rampazzo, Faithful representations for convex Hamilton-Jacobi equations, Preprint, Univ. di Padova.

[30] L. Rifford, Semiconcave control-Lyapunov functions and stabilizing feedbacks, SIAM J. Control Optim. 41 (3) (2002) 659-681.

[31] L. Rifford, Singularities of viscosity solutions and the stabilization problem in the plane, Indiana Univ. Math. J. 52 (5) (2003) $1373-1396$.

[32] M. Tamm, Subanalytic sets in the calculus of variations, Acta Math. 146 (1981).

[33] E. Trélat, Some properties of the value function and its level sets for affine control systems with quadratic cost, J. Dynam. Control Systems 6 (4) (2000) 511-541.

[34] E. Trélat, Etude asymptotique et transcendance de la fonction valeur en contrôle optimal; catégorie log-exp en géométrie sous-Riemannienne dans le cas Martinet, Thèse, Univ. de Bourgogne, 2000.

[35] E. Trélat, Solutions sous-analytiques globales de certaines équations d'Hamilton-Jacobi, C. R. Acad. Sci. Paris, Ser. I 337 (10) (2003) 653656.

[36] L. van den Dries, C. Miller, Geometric categories and o-minimal structures, Duke Math. J. 84 (2) (1996). 